\title{
A Gauge Model for Quantum Mechanics on a Stratified Space
}

\author{
J. Huebschmann ${ }^{1}$, G. Rudolph ${ }^{2}$, M. Schmidt ${ }^{2}$ \\ 1 USTL, UFR de Mathématiques, CNRS-UMR 8524, 59655 Villeneuve d'Ascq Cédex, \\ France. E-mail: Johannes.Huebschmann@math.univ-lille1.fr \\ 2 Institute for Theoretical Physics, University of Leipzig, Augustusplatz 10/11, \\ 04109 Leipzig, Germany
}

Received: 3 April 2007 / Accepted: 15 October 2008

Published online: 9 December 2008 - (C) Springer-Verlag 2008

\begin{abstract}
In the Hamiltonian approach on a single spatial plaquette, we construct a quantum (lattice) gauge theory which incorporates the classical singularities. The reduced phase space is a stratified Kähler space, and we make explicit the requisite singular holomorphic quantization procedure on this space. On the quantum level, this procedure yields a costratified Hilbert space, that is, a Hilbert space together with a system which consists of the subspaces associated with the strata of the reduced phase space and of the corresponding orthoprojectors. The costratified Hilbert space structure reflects the stratification of the reduced phase space. For the special case where the structure group is $\mathrm{SU}(2)$, we discuss the tunneling probabilities between the strata, determine the energy eigenstates and study the corresponding expectation values of the orthoprojectors onto the subspaces associated with the strata in the strong and weak coupling approximations.
\end{abstract}

\section{Contents}

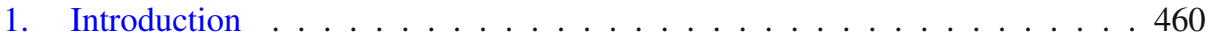

2. The Classical Picture . . . . . . . . . . . . . . . . . . . . . . . . . . 461

2.1 The model . . . . . . . . . . . . . . . . . . . . . . . . . . . . . . 461

2.2 The Kähler structure on the unreduced phase space . . . . . . . . 463

2.3 Symmetry reduction . . . . . . . . . . . . . . . . . . 463

2.4 The stratified Kähler structure on the reduced phase space . . . . . . . 465

3. The Quantum Picture . . . . . . . . . . . . . . . . . . . 467

3.1 Holomorphic quantization . . . . . . . . . . . . . 468

3.2 Schrödinger quantization . . . . . . . . . . . . . . . . . . 470

3.3 The costratified Hilbert space structure . . . . . . . . . . . . . 471

3.4 Observables . . . . . . . . . . . . . . . . . . . . . 472

4. The Costratified Hilbert Space for $\mathrm{SU}(2) \ldots \ldots . \ldots . \ldots 473$

4.1 Group theoretical data . . . . . . . . . . . . . . . . . . 473 
4.2 The costratified Hilbert space structure . . . . . . . . . . . . . . . . . . 474

4.3 Tunneling between strata . . . . . . . . . . . . . . . . . . . . 477

4.4 Adapted orthonormal bases . . . . . . . . . . . . . . . . . . . 478

4.5 Representation in terms of $L^{2}[0, \pi] \ldots \ldots$. . . . . . . . . . . . 479

5. Energy Eigenvalues and Eigenstates for SU(2) . . . . . . . . . . . . . . . 480

5.1 The Hamiltonian . . . . . . . . . . . . . . . . . . . . . . . . 480

5.2 Eigenvalues and eigenstates . . . . . . . . . . . . . . . . 483

6. Expectation Values of the Costratification Orthoprojectors for SU(2) . . . . 485

6.1 Expectation values . . . . . . . . . . . . . . . . . 485

6.2 Strong coupling approximation . . . . . . . . . . . . . . . . 487

6.3 Weak coupling approximation . . . . . . . . . . . . . . . . 488

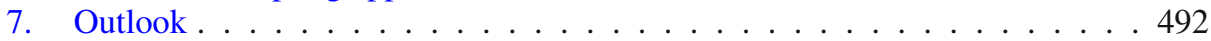

\section{Introduction}

According to DIRAC, the correspondence between a classical theory and its quantum counterpart should be based on an analogy between their mathematical structures. An interesting issue is then that of the role of singularities in quantum problems. Singularities are known to arise in classical phase spaces. For example, in the Hamiltonian picture of a theory, reduction modulo symmetries leads in general to singularities on the classical level. Thus the question arises whether, on the quantum level, there is a suitable structure having the classical singularities as its shadow and whether and how we can uncover it. As far as we know, one of the first papers in this topic is that of EMMRICH AND RÖMER [17]. This paper indicates that wave functions may "congregate" near a singular point, which goes counter to the sometimes quoted statement that singular points in a quantum problem are a set of measure zero so cannot possibly be important. In a similar vein, ASOREY ET AL observed that vacuum nodes correspond to the chiral gauge orbits of reducible gauge fields with non-trivial magnetic monopole components [8]. It is also noteworthy, cf. e.g. [4] and the references there, that in classical mechanics and in classical field theories singularities in the solution spaces are the rule rather than the exception. This is in particular true for Yang-Mills theories and for Einstein's gravitational theory; see for example [5,6].

In [31], one of us isolated a certain class of Kähler spaces with singularities, referred to as stratified Kähler spaces. To explore the potential impact of classical phase space singularities on quantum problems, in [32], he then developed the notion of costratified Hilbert space. This is the appropriate quantum state space over a stratified space; it consists of a system of Hilbert spaces, one for each stratum which arises from quantization on the closure of that stratum. The stratification provides bounded linear operators between these Hilbert spaces reversing the partial ordering among the strata, and these linear operators are compatible with the quantizations. The notion of costratified Hilbert space is, perhaps, the quantum structure which has the classical singularities as its shadow. In [32], the ordinary Kähler quantization scheme has been extended to such a scheme over (complex analytic) stratified Kähler spaces. The appropriate quantum Hilbert space is, in general, a costratified Hilbert space. Examples abound; one such class of examples, involving holomorphic nilpotent orbits and in particular angular momentum zero spaces, has been treated in [32].

Gauge theory in the Hamiltonian approach, phrased on a finite spatial lattice, leads to tractable finite-dimensional models for which one can analyze the role of singularities explicitly. Under such circumstances, after a choice of tree gauge has been 
made, the unreduced classical phase space amounts to the total space $\mathrm{T}^{*}(K \times \cdots \times K)$ of the cotangent bundle on a product of finitely many copies of the manifold underlying the structure group $K$. Gauge transformations are then given by the lift of the action of $K$ on $K \times \cdots \times K$ by diagonal conjugation. This leads to a finite-dimensional Hamiltonian system with symmetries. For first results on the stratified structure of both the reduced configuration space and the reduced phase space of systems of this type, see $[10,11,18,29,30]$. Within canonical quantization for the unreduced system, the algebra of observables and its representations have been extensively investigated, see [40-42] for quantum electrodynamics and $[36,38,39]$ for quantum chromodynamics. However, in this approach, the implementation of singularities is far from being clear.

In the present paper we will consider the case of one copy of $K$. This corresponds to a lattice consisting of a single plaquette. The unreduced phase space $\mathrm{T}^{*} K$ carries an invariant complex structure, and the complex and cotangent bundle symplectic structures combine to give an invariant Kähler structure. Thus, the stratified Kähler quantization scheme of [32] referred to above can be applied. We construct the costratified Hilbert space on the reduced phase space by reduction after quantization. Ordinary half-form Kähler quantization on $\mathrm{T}^{*} K$ yields a Hilbert space of holomorphic and, therefore, continuous wave functions on $\mathrm{T}^{*} K$, and we take the total Hilbert space of our theory to be the subspace of $K$-invariants. Given a stratum, we then consider the space of functions in the Hilbert space which vanish on the stratum, and we take the orthogonal complement of this space as the Hilbert space associated with the stratum. Now, in the Kähler polarization, among the classical observables, only the constants can be quantized directly. However, the holomorphic Peter-Weyl theorem [35] or, equivalently, a version of the Segal-Bargmann transform [25], yields an isomorphism between the total Hilbert space arising from Kähler quantization and the Hilbert space of the Schrödinger representation. Via this isomorphism, the costratified structure passes to the Schrödinger picture. On the other hand, observables defined in the Schrödinger picture via half-form quantization, for example, the Hamiltonian, can be transferred to the holomorphic picture as well. Our approach includes the quantization of arbitrary conjugation invariant Hamiltonian systems on the total space of the cotangent bundle of a compact Lie group. In this paper we concentrate on the particular case of SU(2) with a lattice gauge theoretic Hamiltonian.

The paper is organized as follows. In Sect. 2 we introduce the model and give a brief description of the stratified Kähler structure of its reduced classical phase space. Sect. 3 contains the construction of the costratified Hilbert space structure for general $\operatorname{SU}(n)$. In Sect. 4, we then make this construction explicit for SU(2). In Sect. 5 we determine the energy eigenvalues and eigenstates of our model for SU(2). Finally, in Sect. 6, we discuss the corresponding expectation values of the orthoprojectors onto the subspaces associated with the strata and derive approximations for strong and weak coupling.

\section{The Classical Picture}

2.1. The model. Let $K$ be a compact connected Lie group and let $\mathfrak{k}$ be its Lie algebra. We consider lattice gauge theory with structure group $K$ in the Hamiltonian approach on a single spatial plaquette. By means of a tree gauge, the reduced phase space of the system can be shown to be isomorphic, as a stratified symplectic space, to the reduced

* For an arbitray lattice $\Lambda$, a tree gauge amounts to a choice of maximal tree in $\Lambda$, the parallel transporters along the on-tree links being set equal to the identity of $K$; this leaves the parallel transporters along the off-tree links as variables and constant gauge transformations as symmetries. In our simple example, there is only one off-tree link. 
phase space of the following simpler system. The unreduced configuration space is the group manifold $K$ and gauge transformations are given by the action of $K$ upon itself by inner automorphisms. The unreduced phase space is the cotangent bundle $\mathrm{T}^{*} K$, acted upon by the lifted action. This action is well known to be Hamiltonian and the corresponding momentum mapping $\mu: \mathrm{T}^{*} K \rightarrow \mathfrak{k}^{*}$ is given by a familiar expression [1]. We trivialize $\mathrm{T}^{*} K$ in the following fashion: Endow $\mathfrak{k}$ with an invariant positive definite inner product $\langle\cdot, \cdot\rangle$; we could take, for example, the negative of the Killing form, but this is not necessary. By means of the inner product, we identify $\mathfrak{k}$ with its dual $\mathfrak{k}^{*}$ and the total space T $K$ of the tangent bundle of $K$ with the total space $\mathrm{T}^{*} K$ of the cotangent bundle of $K$. Composing the latter identification with the inverse of left translation we obtain a diffeomorphism

$$
\mathrm{T}^{*} K \rightarrow \mathrm{T} K \rightarrow K \times \mathfrak{k}
$$

It is $K$-bi-invariant w.r.t. the action of $K \times K$ on $K \times \mathfrak{k}$ given by

$$
(x, Y) \mapsto\left(a x b, \operatorname{Ad}_{b^{-1}} Y\right), \quad a, b, x \in K, \quad Y \in \mathfrak{k} .
$$

In the variables $(x, Y) \in K \times \mathfrak{k}$, the lifted action of $K$ reads

$$
(x, Y) \mapsto\left(a x a^{-1}, \operatorname{Ad}_{a} Y\right), \quad a \in K,
$$

and the symplectic potential $\theta: \mathrm{TT}^{*} K \rightarrow \mathbb{R}$ is given by

$$
\theta_{(x, Y)}(x V, W)=\langle Y, V\rangle, \quad V, W \in \mathfrak{k},
$$

where the association $(x, V) \mapsto x V(x \in K, V \in \mathfrak{k})$ refers to left translation in $\mathrm{T} K$. Accordingly, the symplectic form $\omega=-d \theta$ has the explicit description

$$
\omega_{(x, Y)}\left(\left(x V_{1}, W_{1}\right),\left(x V_{2}, W_{2}\right)\right)=\left\langle V_{1}, W_{2}\right\rangle-\left\langle W_{1}, V_{2}\right\rangle+\left\langle Y,\left[V_{1}, V_{2}\right]\right\rangle,
$$

where $V_{1}, V_{2}, W_{1}, W_{2} \in \mathfrak{k}$. The Poisson bracket of functions $f, g \in C^{\infty}(K \times \mathfrak{k})$ is given by

$$
\{f, g\}(x, Y)=\left\langle f_{K}(x, Y), g_{\mathfrak{k}}(x, Y)\right\rangle-\left\langle f_{\mathfrak{k}}(x, Y), g_{K}(x, Y)\right\rangle-\left\langle Y,\left[f_{\mathfrak{k}}(x, Y), g_{\mathfrak{k}}(x, Y)\right]\right\rangle,
$$

where $f_{K}$ and $f_{\mathfrak{k}}$ are $\mathfrak{k}$-valued functions on $K \times \mathfrak{k}$ representing the partial derivatives of $f$ along $K$ and $\mathfrak{k}$, respectively. They are defined by

$$
\left\langle f_{K}(x, Y), Z\right\rangle=\left.\frac{\mathrm{d}}{\mathrm{d} t}\right|_{t=0} f\left(x \mathrm{e}^{t Z}, Y\right), \quad\left\langle f_{\mathfrak{k}}(x, Y), Z\right\rangle=\left.\frac{\mathrm{d}}{\mathrm{d} t}\right|_{t=0} f(x, Y+t Z),
$$

for any $Z \in \mathfrak{k}$. The momentum mapping $\mu$ takes the form

$$
\mu(x, Y)=\operatorname{Ad}_{x} Y-Y, x \in K, Y \in \mathfrak{k} .
$$

In $[10,11,18], \mathrm{T}^{*} K$ has been trivialized by right translation and the sign conventions necessarily differ. The (classical unreduced) Hamiltonian $H: \mathrm{T}^{*} K \rightarrow \mathbb{R}$ of our model is given by

$$
H(x, Y)=\frac{1}{2}|Y|^{2}+\frac{v}{2}(3-\operatorname{Re} \operatorname{tr}(x)), x \in K, Y \in \mathfrak{k} .
$$

Here $|\cdot|$ denotes the norm defined by the inner product on $\mathfrak{k}$, the constant $v$ is defined by $v=1 / g^{2}$, where $g$ is the coupling constant, and the trace refers to some representation; below we will suppose $K$ to be realized as a closed subgroup of some unitary group $\mathrm{U}(n)$. Moreover, we have set the lattice spacing equal to 1 . The Hamiltonian $H$ is manifestly gauge invariant. 
Remark 2.1. Ordinary Yang-Mills theory on $S^{1}$ proceeds by reduction relative to the group of all gauge transformations. As an intermediate step, one can perform reduction relative to the group of based gauge transformations. This procedure provides our unreduced model, i. e., the Hamiltonian $K$-space $\mathrm{T}^{*} K$. Thus, this model recovers a true continuum theory. Starting at the lattice theory on a single plaquette, we have bypassed the reduction relative to the group of based gauge transformations. Our model therefore includes the continuum theory on $S^{1}$ and serves as a building block of a lattice gauge theory as well.

The quantization of Yang-Mills theory on $S^{1}$ in the Hamiltonian approach has been worked out in $[15,16,24,28,44,45,57,58]$. In $[44,45,58]$ the authors proceed through Rieffel induction, starting from the full continuum theory, and arrive at the Hilbert space $L^{2}(K, \mathrm{~d} x)^{K}$ of square-integrable functions on $K$ invariant under inner automorphisms of $K$. See also [43, $\S$ IV.3.7,8] and the references there. We shall arrive at the same Hilbert space almost immediately, as we start at a later stage in the reduction procedure, but this is only a preliminary stage for what we are aiming at: the construction of a costratified Hilbert space to study the role of singularities in the quantum theory.

2.2. The Kähler structure on the unreduced phase space. We recall that a Kähler manifold is a complex manifold, endowed with a positive definite Hermitian form whose imaginary part, necessarily an ordinary real 2-form, is closed and non-degenerate and hence a symplectic structure. Equivalently, a Kähler manifold is a smooth manifold, endowed with a complex and a symplectic structure, and the two structures are required to be compatible. One way of phrasing the compatibility condition is to require that Poisson brackets of holomorphic functions be zero.

The unreduced phase space $\mathrm{T}^{*} K$ acquires a Kähler structure in the following manner: We suppose $K$ realized as a closed subgroup of some unitary group $\mathrm{U}(n)$; then the complexification $K^{\mathbb{C}}$ of $K$ is the complex subgroup of $\operatorname{GL}(n, \mathbb{C})$ generated by $K$. By restriction, the polar decomposition map

$$
\mathrm{U}(n) \times \mathrm{u}(n) \longrightarrow \mathrm{GL}(n, \mathbb{C}), \quad(x, Y) \longmapsto x \mathrm{e}^{i Y},
$$

yields a diffeomorphism

$$
K \times \mathfrak{k} \longrightarrow K^{\mathbb{C}}, \quad(x, Y) \longmapsto x \mathrm{e}^{i Y},
$$

commonly referred to as the polar decomposition of $K^{\mathbb{C}}$. The polar decomposition is manifestly $K$-bi-invariant w.r.t. the action of $K \times K$ on $K \times \mathfrak{k}$ spelled out above. Thus, the composite of the trivialization (2.1) of $\mathrm{T}^{*} K$ with the polar decomposition map (2.6) is a $K$-bi-invariant diffeomorphism $\mathrm{T}^{*} K \rightarrow K^{\mathbb{C}}$. The resulting complex structure on $\mathrm{T}^{*} K \cong K^{\mathbb{C}}$ and the cotangent bundle symplectic structure combine to give a $K$-bi-invariant Kähler structure, having as global Kähler potential the real analytic function $\kappa$ given by

$$
\kappa\left(x \mathrm{e}^{i Y}\right)=|Y|^{2} .
$$

An explicit calculation which justifies this assertion may be found in [25].

2.3. Symmetry reduction. Let $\mathcal{X}$ denote the adjoint quotient $K / \mathrm{Ad}$; this is the reduced configuration space of our model. In the standard manner, we decompose $\mathcal{X}$ as a disjoint union $\mathcal{X}=\bigcup_{\tau, i} \mathcal{X}_{\tau, i}$. Here, $\tau$ ranges over the orbit types of the action, $\mathcal{X}_{\tau}$ denotes the subset of $\mathcal{X}$ which consists of orbits of type $\tau$, and $i$ labels the connected components of 
this subset. We will refer to this decomposition as the orbit type stratification of $\mathcal{X}$. It is a stratification in the sense of e. g. Goresky-MacPherson [20]. For our purposes it suffices to know that it is a manifold decomposition in the ordinary sense, i. e., the $\mathcal{X}_{\tau, i}$ are manifolds and the frontier condition holds, viz. $\mathcal{X}_{\tau_{1}, i_{1}} \subseteq \overline{\mathcal{X}_{\tau_{2}, i_{2}}}$ whenever $\mathcal{X}_{\tau_{1}, i_{1}} \cap \overline{\mathcal{X}_{\tau_{2}, i_{2}}} \neq \emptyset$. An explicit description of $\mathcal{X}$ arises from a choice of a maximal toral subgroup $T \subseteq K$. Let $W$ be the Weyl group of $K$. It is well known that the inclusion $T \hookrightarrow K$ induces a homeomorphism from the orbit space $T / W$ onto the quotient $\mathcal{X}=K /$ Ad which identifies orbit type strata.

The reduced phase space of our model is the zero momentum reduced space $\mu^{-1}(0) / K$ obtained by singular Marsden-Weinstein reduction. We denote this space by $\mathcal{P}$. It acquires a stratified symplectic structure where, similarly to the reduced configuration space $\mathcal{X}$, the stratification is given by the connected components of the orbit type subsets, viz. $\mathcal{P}=\bigcup_{\tau, i} \mathcal{P}_{\tau, i}$. An explicit description of $\mathcal{P}$ is obtained as follows. Let $\mathfrak{t} \subseteq \mathfrak{k}$ be the Lie algebra of $T$. Given $(x, Y) \in K \times \mathfrak{k}$, according to (2.4), the vanishing of $\mu(x, Y)$ implies that $x$ and $Y$ commute. Hence, the pair $(x, Y)$ is conjugate to an element of $T \times \mathfrak{t}$ and the injection $T \times \mathfrak{t} \hookrightarrow K \times \mathfrak{k}$ induces a homeomorphism of $\mathcal{P}$ onto the quotient $(T \times \mathfrak{t}) / W$, where $W$ acts simultaneously on $T$ and $\mathfrak{t}$. This homeomorphism identifies orbit type strata.

In the case $K=\mathrm{SU}(n)$, the torus $T$ can be chosen as the subgroup of diagonal matrices in $K$. Then $t$ is the subalgebra of diagonal matrices in $\mathfrak{k}$. The Weyl group $W$ is the symmetric group $S_{n}$ on $n$ letters, acting on $T$ and $\mathfrak{t}$ by permutation of entries. The reduced configuration space $\mathcal{X} \cong T / W$ amounts to an $(n-1)$-simplex and the orbit type strata correspond to its (open) subsimplices. In particular, the orbit types are labelled by partitions $n=n_{1}+\cdots+n_{k}$ of $n$, where the $n_{i}$ 's are positive integers reflecting the multiplicities of the entries of the elements of $T$. Concerning the reduced phase space $\mathcal{P}$, the orbit types of the action of $W$ on $T \times \mathfrak{t}$ are given by partitions of $n$ again, where the $n_{i}$ 's now are the dimensions of the common eigenspaces of pairs in $T \times \mathfrak{t}$.

For later use, we shall describe $\mathcal{X}$ and $\mathcal{P}$ for $K=\mathrm{SU}(2)$ in detail. Here, $T$ amounts to the complex unit circle and $t$ to the imaginary axis. Then the Weyl group $W=S_{2}$ acts on $T$ by complex conjugation and on $t$ by reflection. Hence, the reduced configuration space $\mathcal{X} \cong T / W$ is homeomorphic to a closed interval and the reduced phase space $\mathcal{P} \cong(T \times \mathfrak{t}) / W$ is homeomorphic to the well-known canoe, see Fig. 1. Corresponding to the partitions $2=2$ and $2=1+1$, there are two orbit types. We denote them by 0 and 1 , respectively. The orbit type subset $\mathcal{X}_{0}$ consists of the classes of $\pm \mathbb{1}$, i. e., of the endpoints of the interval; it decomposes into the connected components $\mathcal{X}_{+}$, consisting of the class of $\mathbb{1}$, and $\mathcal{X}_{-}$, consisting of the class of $-\mathbb{1}$. The orbit type subset $\mathcal{X}_{1}$ is connected and consists of the remaining classes, i. e., of the interior of the interval. The orbit type subset $\mathcal{P}_{0}$ consists of the classes of $( \pm \mathbb{1}, 0)$, i. e., of the vertices of the canoe;

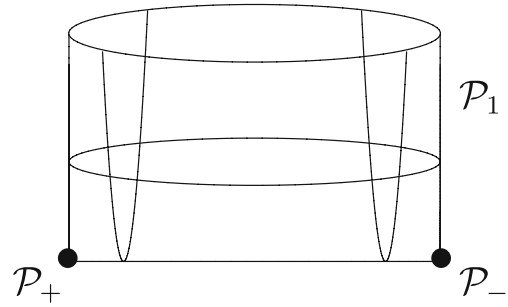

Fig. 1. The reduced phase space $\mathcal{P}$ for $K=\mathrm{SU}(2)$ 
it decomposes into the connected components $\mathcal{P}_{+}$, consisting of the class of $(\mathbb{1}, 0)$, and $\mathcal{P}_{-}$, consisting of the class of $(-\mathbb{1}, 0)$. The orbit type subset $\mathcal{P}_{1}$ consists of the remaining classes, has dimension 2 and is connected.

Remark 2.2. In the case $K=\mathrm{SU}(2)$, as a stratified symplectic space, $\mathcal{P}$ is isomorphic to the reduced phase space of a spherical pendulum, reduced at vertical angular momentum 0 (whence the pendulum is constrained to move in a plane), see [13].

In [31], the notion of stratified Kähler space has been introduced and it has been shown that, under more general circumstances, the Kähler structure on $\mathrm{T}^{*} K \cong K^{\mathbb{C}}$ explained in Subsect. 2.2 descends to a stratified Kähler structure on $\mathcal{P}$ which is compatible with the stratified symplectic structure. A detailed discussion of this stratified Kähler structure can be found in $[33,34]$. For completeness, we include a brief description in Subsect. 2.4 below. Since this will not be needed for quantization, the reader who is interested in the quantum theory only may skip this subsection.

\subsection{The stratified Kähler structure on the reduced phase space. The Weyl group W} acts on $\mathrm{T}^{*} T$ by pull back and on $T^{\mathbb{C}}$ by permutation of entries. The trivialization (2.1) and the polar decomposition (2.6) combine to a $W$-equivariant diffeomorphism

$$
\mathrm{T}^{*} T \rightarrow T \times \mathfrak{t} \rightarrow T^{\mathbb{C}}
$$

This diffeomorphism, in turn, induces a homeomorphism between $\mathcal{P}$ and the quotients $\mathrm{T}^{*} T / W \cong T^{\mathbb{C}} / W$. Moreover, as explained in Subsect. 2.2, the symplectic structure of $\mathrm{T}^{*} T$ and the complex structure of $T^{\mathbb{C}}$ combine to give a Kähler structure on $\mathrm{T}^{*} T \cong T^{\mathbb{C}}$. In the sequel, we shall stick to the notation $T^{\mathbb{C}}$.

Viewed as the orbit space $\mathrm{T}^{*} T / W, \mathcal{P}$ inherits a stratified symplectic structure by singular Marsden-Weinstein reduction. That is to say: (i) The algebra $C^{\infty}\left(T^{\mathbb{C}}\right)^{W}$ of ordinary smooth $W$-invariant functions on $T^{\mathbb{C}}$ inherits a Poisson bracket and thus yields a Poisson algebra of continuous functions on $\mathcal{P} \cong T^{\mathbb{C}} / W$, (ii) for each stratum, the Poisson structure yields an ordinary symplectic Poisson structure on that stratum, and (iii) the restriction mapping from $C^{\infty}\left(T^{\mathbb{C}}\right)^{W}$ to the algebra of ordinary smooth functions on that stratum is a Poisson map.

Viewed as the orbit space $T^{\mathbb{C}} / W, \mathcal{P}$ acquires a complex analytic structure in the standard fashion. The complex structure and the Poisson structure combine to give a stratified Kähler structure on $\mathcal{P}[31,33,34]$. Here the precise meaning of the term "stratified Kähler structure" is that the Poisson structure satisfies (ii) and (iii) above and that the Poisson and complex structures satisfy the additional compatibility requirement that for each stratum, necessarily a complex manifold, the symplectic and complex structures on that stratum combine to give an ordinary Kähler structure.

In the case $K=\mathrm{SU}(n)$, the complex analytic structure admits the following elementary description: Let $\operatorname{Diag}(n, \mathbb{C})$ be the group of diagonal matrices in the full linear group $\operatorname{GL}(n, \mathbb{C})$. The Weyl group $W$ acts on $\operatorname{Diag}(n, \mathbb{C})$ by permutation of entries and the injection of $T^{\mathbb{C}}$ into $\operatorname{Diag}(n, \mathbb{C})$ is compatible with this action. The $n$ elementary symmetric functions $\sigma_{1}, \ldots, \sigma_{n}$ furnish a map

$$
\left(\sigma_{1}, \ldots, \sigma_{n}\right): \operatorname{Diag}(n, \mathbb{C}) \longrightarrow \mathbb{C}^{n}
$$

into complex $n$-space $\mathbb{C}^{n}$. The restriction

$$
\left(\sigma_{1}, \ldots, \sigma_{n-1}\right): T^{\mathbb{C}} \longrightarrow \mathbb{C}^{n-1}
$$


of that map to $T^{\mathbb{C}}$ identifies the orbit space $\mathcal{P} \cong T^{\mathbb{C}} / W$ with the affine subspace of $\mathbb{C}^{n}$ given by the equation $\sigma_{n}=1$ which, in turn, may be identified with a copy of $\mathbb{C}^{n-1}$. In this way, $\mathcal{P}$ inherits an obvious complex structure. Thus, the affine complex $n$-space $\mathbb{C}^{n}$ appears here as the space of normalized complex degree $n$ polynomials, and the orbit space $T^{\mathbb{C}} / W$ amounts to the subspace of normalized complex degree $n$ polynomials with constant coefficient equal to 1 . Indeed, a normalized degree $n$ polynomial $p(z)=z^{n}+a_{1} z^{n-1}+\cdots+a_{n-1} z+a_{n}$ decomposes into its linear factors $p(z)=\prod_{j}\left(z-z_{j}\right)$, and the coefficients $a_{j}$ are given by

$$
a_{j}=(-1)^{j} \sigma_{j}\left(z_{1}, \ldots, z_{n}\right), 1 \leq j \leq n ;
$$

up to the signs $(-1)^{j}$, the map $\sigma$ may thus be viewed as that which sends the $n$-tuple $z_{1}, \ldots, z_{n}$ to the unique normalized degree $n$ polynomial having $z_{1}, \ldots, z_{n}$ as its zeros, the coefficients of degree $\leq n-1$ being taken as coordinates on the space of polynomials. A more profound analysis shows that, indeed, in terms of $\operatorname{SL}(n, \mathbb{C})$ and $\operatorname{GL}(n, \mathbb{C})$, the passage to the quotient (which is here realized via the map (2.8)) amounts to the assignment to a matrix in $\operatorname{SL}(n, \mathbb{C})($ or $\operatorname{GL}(n, \mathbb{C}))$ of its characteristic polynomial.

We shall now describe the stratified Kähler structure on $\mathcal{P}$ explicitly for $K=\mathrm{SU}(2)$. Here, $T^{\mathbb{C}}$ consists of the diagonal matrices $\operatorname{diag}\left(z, z^{-1}\right)$, where $z \in \mathbb{C}^{*}$. The non-trivial element of $W$ interchanges $z$ and $z^{-1}$. To determine the complex structure we note that the map (2.8) is given by the restriction of the first elementary symmetric function $\sigma_{1}$ on $\operatorname{Diag}(2, \mathbb{C})$ to the subgroup $T^{\mathbb{C}}$, i.e.,

$$
\sigma_{1}: T^{\mathbb{C}} \longrightarrow \mathbb{C}, \quad \sigma_{1}\left(\operatorname{diag}\left(z, z^{-1}\right)\right)=z+z^{-1}
$$

this map identifies $T^{\mathbb{C}} / W \cong \mathcal{P}$ with a copy of $\mathbb{C}$ and thus provides a holomorphic coordinate on $\mathcal{P}$. In particular, topologically, the canoe shown in Fig. 1 is just an ordinary plane.

To arrive at a description of the Poisson algebra $C^{\infty}\left(T^{\mathbb{C}}\right)^{W}$, we recall that, once a choice of finitely many generators, say $p$, for the algebra $\mathbb{R}\left[T^{\mathbb{C}}\right]^{W}$ of real $W$-invariant polynomials on $T^{\mathbb{C}}$ has been made, the resulting Hilbert map induces a homeomorphism from $T^{\mathbb{C}} / W \cong \mathcal{P}$ onto a semi-algebraic subset of $\mathbb{R}^{p}$. According to a theorem in [50], any element of $C^{\infty}\left(T^{\mathbb{C}}\right)^{W}$ can be written as a smooth function in these generators. Hence, to describe the Poisson algebra $C^{\infty}\left(T^{\mathbb{C}}\right)^{W}$ it suffices to list the Poisson brackets of these generators. In the case at hand, a set of generators for $\mathbb{R}\left[T^{\mathbb{C}}\right]^{W}$ can be obtained as follows. The complexification $\mathbb{R}\left[T^{\mathbb{C}}\right]_{\mathbb{C}}$ of $\mathbb{R}\left[T^{\mathbb{C}}\right]$ is generated by $z, z^{-1}, \bar{z}, \bar{z}^{-1}$. Since the non-trivial element of $W$ interchanges $z$ and $z^{-1}$ as well as $\bar{z}$ and $\bar{z}^{-1}$, the subalgebra $\mathbb{R}\left[T^{\mathbb{C}}\right]_{\mathbb{C}}^{W}$ of $W$-invariants is generated by the three elementary bisymmetric functions:

$$
\sigma_{1}=z+z^{-1}, \bar{\sigma}_{1}=\bar{z}+\bar{z}^{-1}, \sigma=z \bar{z}^{-1}+\bar{z} z^{-1},
$$

and this algebra may be identified with the complexification of $\mathbb{R}\left[T^{\mathbb{C}}\right]^{W}$ in an obvious manner. These generators are subject to the single defining relation

$$
\left(\sigma_{1}^{2}-4\right)\left(\bar{\sigma}_{1}^{2}-4\right)=\left(\sigma_{1} \bar{\sigma}_{1}-2 \sigma\right)^{2},
$$

see [33]. Hence, $\mathbb{R}\left[T^{\mathbb{C}}\right]^{W}$ is generated by the three real functions $X, Y$ and $\sigma$, where $\sigma_{1}=X+\mathrm{i} Y$. For convenience, instead of $\sigma$, we use $\tau=\frac{2-\sigma}{4}$. In view of (2.10), the generators $X, Y, \tau$ are subject to the relation

$$
Y^{2}=\left(X^{2}+Y^{2}+4(\tau-1)\right) \tau .
$$


In terms of the real coordinates $x$ and $y$ on $T^{\mathbb{C}} \cong \mathbb{C}^{*}$ defined by $z=x+\mathrm{i} y$,

$$
X=x+\frac{x}{r^{2}}, \quad Y=y-\frac{y}{r^{2}}, \quad \tau=\frac{y^{2}}{r^{2}},
$$

where $r^{2}=x^{2}+y^{2}$. The obvious inequality $\tau \geq 0$ brings the semialgebraic nature of the quotient $\mathbb{T}^{\mathbb{C}} / W$ to the fore. To determine the Poisson brackets among the generators $X, Y$ and $\tau$, we recall that, in terms of the coordinates $x$ and $y$, the symplectic structure on $T^{\mathbb{C}} \cong \mathbb{C}^{*}$ is given by $\frac{1}{r^{2}} \mathrm{~d} x \wedge \mathrm{d} y$ whence

$$
\{x, y\}=r^{2} \text {. }
$$

A straightforward calculation involving (2.12) yields the Poisson brackets

$$
\{X, Y\}=X^{2}+Y^{2}+4(2 \tau-1), \quad\{X, \tau\}=2(1-\tau) Y, \quad\{Y, \tau\}=2 \tau X .
$$

The Poisson structure vanishes at the two points $(X, Y)=(2,0)$ and $(X, Y)=(-2,0)$ representing the orbit type strata $\mathcal{P}_{+}$and $\mathcal{P}_{-}$, respectively. Hence, the resulting complex algebraic stratified Kähler structure on $\mathcal{P}$ is singular at these two points. Furthermore, solving (2.11) for $\tau$, we obtain

$$
\tau=\frac{1}{2} \sqrt{Y^{2}+\frac{\left(X^{2}+Y^{2}-4\right)^{2}}{16}}-\frac{X^{2}+Y^{2}-4}{8},
$$

whence, at $(X, Y)=( \pm 2,0), \tau$ is not smooth as a function of the variables $X$ and $Y$. Away from these two points, i.e., on the principal stratum $\mathcal{P}_{1}$, the Poisson structure is symplectic. We refer to the stratified Kähler space under discussion as the exotic plane with two vertices. More details and, in particular, an interpretation in terms of discriminant varieties, may be found in [34].

Remark 2.3. The algebra $\mathbb{R}\left[T^{\mathbb{C}}\right]^{W}$ is the real coordinate ring of $T^{\mathbb{C}} / W$, viewed as a real semi-algebraic set. Similarly, for the description of the Poisson structure on $\mathcal{P}$ we could have used a set of generators of, e.g., the algebra $\mathbb{R}[T \times \mathfrak{t}]^{W}$ of real $W$-invariant polynomials on $T \times \mathfrak{t}$. This is the real coordinate ring of $(T \times \mathfrak{t}) / W$, viewed, in turn, as a semi-algebraic set. Since the diffeomorphism $T \times \mathfrak{t} \cong T^{\mathbb{C}}$ is not algebraic, $\mathbb{R}\left[T^{\mathbb{C}}\right]^{W}$ and $\mathbb{R}[T \times \mathfrak{t}]^{W}$ correspond to different subalgebras of the Poisson algebra $C^{\infty}\left(T^{\mathbb{C}}\right)^{W}$ defining the Poisson structure on $\mathcal{P} \cong T^{\mathbb{C}} / W$.

\section{The Quantum Picture}

Our aim is to push further, in the context of stratified spaces, the ideas which underlie the program of geometric quantization. As our physical Hilbert space we take a certain space of square-integrable holomorphic functions which arises by Kähler quantization $[51,56]$. Through an analogue of the Peter-Weyl theorem, this space is related with the physical Hilbert space arising by ordinary Schrödinger quantization on $K$. Within this Hilbert space we construct the additional structure of a costratification. Thereafter, we discuss observables. 
3.1. Holomorphic quantization. Let $\varepsilon$ be the symplectic (or Liouville) volume form on $\mathrm{T}^{*} K \cong K^{\mathbb{C}}$. In terms of the polar decomposition (2.6), we then have the identity $\varepsilon=d x d Y$, where $d x$ is the volume form on $K$ yielding Haar measure, normalized so that it coincides with the Riemannian volume measure on $K$, and where $d Y$ is the form inducing Lebesgue measure on $\mathfrak{k}$, normalized by the inner product on $\mathfrak{k}$. Next, let $\eta$ be the real $K$-bi-invariant analytic function on $K \times \mathfrak{k} \cong K^{\mathbb{C}}$ defined by

$$
\eta\left(x \mathrm{e}^{i Y}\right)=\sqrt{\operatorname{det}\left(\frac{\sin (\operatorname{ad}(Y))}{\operatorname{ad}(Y)}\right)}, x \in K, Y \in \mathfrak{k},
$$

the square root being the positive one. We note that $\eta^{2}$ is the density of Haar measure on $K^{\mathbb{C}}$ relative to Liouville measure $\varepsilon$, cf. [23] (Lemma 5). To express $\eta$ in terms of a root system, we choose a dominant Weyl chamber in the Cartan subalgebra $\mathfrak{t}$ of $\mathfrak{k}$ and denote by $R^{+}$the corresponding set of positive roots. Then, on $T \times \mathfrak{t} \cong T^{\mathbb{C}}, \eta$ is given by

$$
\eta\left(x \mathrm{e}^{i Y}\right)=\prod_{\alpha \in R^{+}} \frac{\sinh (\alpha(Y))}{\alpha(Y)}, \quad x \in T, Y \in \mathfrak{t},
$$

cf. [25] (2.10). Here the $\alpha$ 's are the real roots, given by $-\mathrm{i}$ times the ordinary complex roots. Let $\kappa$ be the $K$-bi-invariant real analytic function on $K \times \mathfrak{k} \cong K^{\mathbb{C}}$ defined by (2.7). Half-form Kähler quantization on $K^{\mathbb{C}}$ yields the Hilbert space $\mathcal{H} L^{2}\left(K^{\mathbb{C}}, \mathrm{e}^{-\kappa / \hbar} \eta \varepsilon\right)$ of holomorphic functions on $K^{\mathbb{C}}$ which are square-integrable relative to the measure $\mathrm{e}^{-\kappa / \hbar} \eta \varepsilon[25]$. The scalar product is given by

$$
\left\langle\psi_{1}, \psi_{2}\right\rangle=\frac{1}{\operatorname{vol}(K)} \int_{K^{\mathbb{C}}} \overline{\psi_{1}} \psi_{2} \mathrm{e}^{-\kappa / \hbar} \eta \varepsilon
$$

For our purpose there is no need to write down the relevant half-forms explicitly. They are subsumed under the measure.

Left and right translation turn the Hilbert space $\mathcal{H} L^{2}\left(K^{\mathbb{C}}, \mathrm{e}^{-\kappa / \hbar} \eta \varepsilon\right)$ into a unitary representation of $K \times K$. The Hilbert space associated with $\mathcal{P}$ by reduction after quantization is the subspace $\mathcal{H} L^{2}\left(K^{\mathbb{C}}, \mathrm{e}^{-\kappa / \hbar} \eta \varepsilon\right)^{K}$ of $K$-invariants relative to conjugation.

We will now describe the Hilbert space $\mathcal{H} L^{2}\left(K^{\mathbb{C}}, \mathrm{e}^{-\kappa / \hbar} \eta \varepsilon\right)^{K}$ as a Hilbert space of $W$-invariant holomorphic functions on $T^{\mathbb{C}}$ that are square-integrable relative to a measure of the kind $\mathrm{e}^{-\kappa / \hbar} \gamma \varepsilon_{T}$ for a suitable density function $\gamma$ on $T^{\mathbb{C}}$, where $\varepsilon_{T}$ denotes the Liouville volume form on $T^{\mathbb{C}} \cong \mathrm{T}^{*} T$. This Hilbert space may in fact be viewed as coming from quantization after reduction, i. e., by quantization on $T^{\mathbb{C}} / W$. Here and below we do not distinguish in notation between the function $\mathrm{e}^{-\kappa / \hbar}$ defined on $K^{\mathbb{C}}$ and its restriction to $T^{\mathbb{C}}$.

Let $m=\operatorname{dim} K$ and $r=\operatorname{dim} T$. To construct the function $\gamma$, consider the conjugation mapping

$$
q^{\mathbb{C}}:\left(K^{\mathbb{C}} / T^{\mathbb{C}}\right) \times T^{\mathbb{C}} \longrightarrow K^{\mathbb{C}},\left(y T^{\mathbb{C}}, t\right) \mapsto y t y^{-1}, y \in K^{\mathbb{C}}, t \in T^{\mathbb{C}},
$$

and integrate the induced $(2 m)$-form $\left(q^{\mathbb{C}}\right)^{*}\left(\mathrm{e}^{-\kappa / \hbar} \eta \varepsilon\right)$ over "the fibers" $K^{\mathbb{C}} / T^{\mathbb{C}}$. Although the fibers are non-compact, in view of the Gaussian constituent $\mathrm{e}^{-\kappa / \hbar}$, this 
integration is a well defined operation. Let $\widetilde{\gamma}$ be the density of the resulting $(2 r)$-form on $T^{\mathbb{C}}$ relative to the Liouville volume form $\varepsilon_{T}$ on $T^{\mathbb{C}} \cong \mathrm{T}^{*} T$, and let

$$
\gamma=\frac{\tilde{\gamma}}{|W| \mathrm{e}^{-\kappa / \hbar}}
$$

where $|W|$ is the order of the Weyl group. An explicit calculation of $\gamma$ can be found in Theorem 3 of Sect. 2 of [19], see also Theorem 12 in [26]. The following is the analogue of Weyl's integration formula, spelled out for $\operatorname{Ad}(K)$-invariant holomorphic functions.

Proposition 3.1. Given two holomorphic $\operatorname{Ad}(K)$-invariant functions $\psi_{1}, \psi_{2}$ on $K^{\mathbb{C}}$ that are square-integrable relative to the measure $\mathrm{e}^{-\kappa / \hbar} \eta \varepsilon$,

$$
\int_{K^{\mathbb{C}}} \overline{\psi_{1}} \psi_{2} \mathrm{e}^{-\kappa / \hbar} \eta \varepsilon=\int_{T^{\mathbb{C}}} \overline{\psi_{1}} \psi_{2} \mathrm{e}^{-\kappa / \hbar} \gamma \varepsilon_{T}
$$

Proof. Since $\psi_{1}$ and $\psi_{2}$ are $\operatorname{Ad}(K)$-invariant and holomorphic, they are $\operatorname{Ad}\left(K^{\mathbb{C}}\right)$ invariant. Hence, their pullbacks under the conjugation mapping (3.2) are constant along the constituent $K^{\mathbb{C}} / T^{\mathbb{C}}$. Since the conjugation mapping has degree equal to the order $|W|$ of the Weyl group and since the complement of the image under the conjugation mapping has measure zero,

$$
\int_{K^{\mathbb{C}}} \overline{\psi_{1}} \psi_{2} \mathrm{e}^{-\kappa / \hbar} \eta \varepsilon=\frac{1}{|W|} \int_{T^{\mathbb{C}}} \overline{\psi_{1}} \psi_{2} \tilde{\gamma} \varepsilon_{T}=\int_{T^{\mathbb{C}}} \overline{\psi_{1}} \psi_{2} \mathrm{e}^{-\kappa / \hbar} \gamma \varepsilon_{T} .
$$

The proposition implies that the restriction mapping induces an isomorphism

$$
\mathcal{H} L^{2}\left(K^{\mathbb{C}}, \mathrm{e}^{-\kappa / \hbar} \eta \varepsilon\right)^{K} \longrightarrow \mathcal{H} L^{2}\left(T^{\mathbb{C}}, \mathrm{e}^{-\kappa / \hbar} \gamma \varepsilon_{T}\right)^{W}
$$

of Hilbert spaces where, according to (3.1), the scalar product in $\mathcal{H} L^{2}\left(T^{\mathbb{C}}, \mathrm{e}^{-\kappa / \hbar} \gamma \varepsilon_{T}\right)^{W}$ is given by

$$
\frac{1}{\operatorname{vol}(K)} \int_{T^{\mathbb{C}}} \overline{\psi_{1}} \psi_{2} e^{-\kappa / \hbar} \gamma \varepsilon_{T}
$$

A basis of $\mathcal{H} L^{2}\left(K^{\mathbb{C}}, \mathrm{e}^{-\kappa / \hbar} \eta \varepsilon\right)^{K}$ and hence of $\mathcal{H} L^{2}\left(T^{\mathbb{C}}, \mathrm{e}^{-\kappa / \hbar} \gamma \varepsilon_{T}\right)^{W}$ is obtained as follows. For a highest weight $\lambda$ relative to the chosen dominant Weyl chamber, we will denote by $\chi_{\lambda}^{\mathbb{C}}$ the irreducible character of $K^{\mathbb{C}}$ associated with $\lambda$. The holomorphic Peter-Weyl theorem established in [35], see Remark 3.1 below for historical comments, implies that the total Hilbert space $\mathcal{H}$ contains the complex vector space which underlies the algebra $\mathbb{C}\left[K^{\mathbb{C}}\right]^{K}$ of $\operatorname{Ad}(K)$-invariant polynomial functions on $K^{\mathbb{C}}$ as a dense subspace. Hence the irreducible characters $\chi_{\lambda}^{\mathbb{C}}$ of $K^{\mathbb{C}}$ form a basis of $\mathcal{H} L^{2}\left(K^{\mathbb{C}}, \mathrm{e}^{-\kappa / \hbar} \eta \varepsilon\right)^{K}$. 
3.2. Schrödinger quantization. Half-form Schrödinger quantization on $\mathrm{T}^{*} K$ yields the Hilbert space $L^{2}(K, \mathrm{~d} x)$ of ordinary square-integrable functions on $K$ [25] with scalar product

$$
\left\langle\psi_{1}, \psi_{2}\right\rangle=\frac{1}{\operatorname{vol}(K)} \int_{K} \overline{\psi_{1}} \psi_{2} \mathrm{~d} x .
$$

We remind the reader that for reasons explained above we have normalized the Haar measure on $K$ so that it coincides with the Riemannian volume measure. Left and right translation turn the Hilbert space $L^{2}(K, \mathrm{~d} x)$ into a unitary $(K \times K)$-representation. The Hilbert space associated with $\mathcal{P}$ by reduction after quantization is the subspace $L^{2}(K, \mathrm{~d} x)^{K}$ of $K$-invariants. It also arises as the physical Hilbert space of the observable algebra [39] and by quantization via Rieffel induction [44,45,57,58], see also $[43, \S \S I V .3 .7,8]$.

Similarly as $\mathcal{H} L^{2}\left(K^{\mathbb{C}}, \mathrm{e}^{-\kappa / \hbar} \eta \varepsilon\right)^{K}$, the space $L^{2}(K, \mathrm{~d} x)^{K}$ can alternatively be viewed as a Hilbert space of $W$-invariant functions which now live on $T$ rather than on $T^{\mathbb{C}}$. Indeed, let $v: T \rightarrow \mathbb{R}$ be the real function given by $v(t)=\operatorname{vol}(\operatorname{Ad}(K) t) /|W|, t \in T$, that is, $v(t)$ is the Riemannian volume of the conjugacy class $\operatorname{Ad}(K) t$ in $K$ generated by $t \in T$, divided by the order $|W|$ of the Weyl group. Restriction of $\operatorname{Ad}(K)$-invariant functions from $K$ to $T$ is well known to induce an isomorphism

$$
L^{2}(K, d x)^{K} \longrightarrow L^{2}(T, v \mathrm{~d} t)^{W}
$$

of Hilbert spaces where the scalar product on $L^{2}(T, v d t)^{W}$ is given by

$$
\frac{1}{\operatorname{vol}(K)} \int_{T} \overline{\psi_{1}} \psi_{2} v \mathrm{~d} t
$$

Given a highest weight $\lambda$, we will denote by $\chi_{\lambda}$ the corresponding irreducible character of $K$, so that $\chi_{\lambda}$ is the restriction of $\chi_{\lambda}^{\mathbb{C}}$ to $K$. The $\chi_{\lambda}$ 's form an orthonormal basis of $L^{2}(K, \mathrm{~d} x)^{K}$.

Let $\rho=1 / 2 \sum_{\alpha \in R^{+}} \alpha$ denote the half sum of the positive roots and let $C_{\lambda}$ be the constant

$$
C_{\lambda}=(\hbar \pi)^{\operatorname{dim}(K) / 2} \mathrm{e}^{\hbar|\lambda+\rho|^{2}},
$$

where $|\lambda+\rho|$ refers to the norm of $\lambda+\rho$ relative to the inner product on $\mathfrak{k}$.

Theorem 3.2. The assignment to $\chi_{\lambda}$ of $C_{\lambda}^{-1 / 2} \chi_{\lambda}^{\mathbb{C}}$, as $\lambda$ ranges over the highest weights, yields a unitary isomorphism

$$
L^{2}(K, \mathrm{~d} x)^{K} \longrightarrow \mathcal{H} L^{2}\left(K^{\mathbb{C}}, \mathrm{e}^{-\kappa / \hbar} \eta \varepsilon\right)^{K}
$$

of Hilbert spaces.

Proof. The holomorphic function $C_{\lambda}^{-1 / 2} \chi_{\lambda}^{\mathbb{C}}$ is the image of $\chi_{\lambda}$ under the Segal-Bargmann transform

$$
L^{2}(K, \mathrm{~d} x) \longrightarrow \mathcal{H} L^{2}\left(K^{\mathbb{C}}, \mathrm{e}^{-\kappa / \hbar} \eta \varepsilon\right)
$$

which is a unitary isomorphism [21]. The assertion follows because $\chi_{\lambda}$ and $\chi_{\lambda}^{\mathbb{C}}$ are bases in $L^{2}(K, \mathrm{~d} x)^{K}$ and $\mathcal{H} L^{2}\left(K^{\mathbb{C}}, \mathrm{e}^{-\kappa / \hbar} \eta \varepsilon\right)^{K}$, respectively. Alternatively, the assertion is a direct consequence of Theorem 5.3 in [35]. 
Remark 3.1. The Segal-Bargmann transform (3.11) and, therefore, the isomorphism (3.10), rely on the description of the Hilbert spaces $L^{2}(K, d x)$ and $\mathcal{H} L^{2}\left(K^{\mathbb{C}}, \mathrm{e}^{-\kappa / \hbar} \eta \varepsilon\right)$ as half-form Hilbert spaces and involve the appropriate metaplectic correction [56].

Originally, in [21], see also [22], the Segal-Bargmann transform was developed via heat kernel analysis on $K$ and $K^{\mathbb{C}}$. More recently, an alternative purely geometric description of this transform in terms of representative functions and independent of heat kernel analysis has been given in Theorem 5.3 of [35]. This description relies on the holomorphic Peter-Weyl theorem [35]. The holomorphic Peter-Weyl theorem yields a proof of Theorem 3.2 above as well and the geometric methods in [35] also recover the heat kernel analysis. On the other hand, the holomorphic Peter-Weyl theorem can likewise be deduced from the Segal-Bargmann transform developed in [25], combined with the ordinary Peter-Weyl theorem.

Alternatively, we can describe the isomorphism (3.11) as being induced by the corresponding BKS-pairing map from $L^{2}(K, \mathrm{~d} x)$ to $\mathcal{H} L^{2}\left(K^{\mathbb{C}}, \mathrm{e}^{-\kappa / \hbar} \eta \varepsilon\right)$, multiplied by a factor $(4 \pi)^{-\operatorname{dim}(K) / 4}$. For details, see [25] (description in terms of the heat kernel on $K$ ) or Theorem 6.5 in [35] (description in terms of representative functions).

Theorem 3.2 entails that the complex characters $\chi_{\lambda}^{\mathbb{C}}$ satisfy the orthogonality relations

$$
\left\langle\chi_{\lambda}^{\mathbb{C}}, \chi_{\lambda^{\prime}}^{\mathbb{C}}\right\rangle=C_{\lambda} \delta_{\lambda \lambda^{\prime}}
$$

Hence, the vectors $C_{\lambda}^{-1 / 2} \chi_{\lambda}^{\mathbb{C}}$, where $\lambda$ ranges over the highest weights, form an orthonormal basis of $\mathcal{H} L^{2}\left(K^{\mathbb{C}}, \mathrm{e}^{-\kappa / \hbar} \eta \varepsilon\right)^{K}$.

From now on, we will take the Hilbert space of our model to be the Hilbert space $\mathcal{H}$ with orthonormal basis $|\lambda\rangle$ labelled by the highest weights. In the holomorphic representation, $\mathcal{H}$ is then realized as $\mathcal{H} L^{2}\left(K^{\mathbb{C}}, \mathrm{e}^{-\kappa / \hbar} \eta \varepsilon\right)^{K}$ or, equivalently, as

$$
\mathcal{H} L^{2}\left(T^{\mathbb{C}}, \mathrm{e}^{-\kappa / \hbar} \gamma \varepsilon_{T}\right)^{W}
$$

whereas, in the Schrödinger representation, $\mathcal{H}$ is realized as $L^{2}(K, \mathrm{~d} x)^{K}$ or, equivalently, as $L^{2}(T, v \mathrm{~d} t)^{W}$. The passage to the respective representation is achieved by substitution for $|\lambda\rangle$ of the function $C_{\lambda}^{-1 / 2} \chi_{\lambda}^{\mathbb{C}}$ or $\chi_{\lambda}$ as appropriate.

3.3. The costratified Hilbert space structure. We will now construct the additional structure of a costratification. To begin with, we recall from [31] the precise definition of a costratified Hilbert space. Let $N$ be a stratified space. Let $\mathcal{C}_{N}$ be the category whose objects are the strata of $N$ and whose morphisms are the inclusions $Y^{\prime} \subseteq \bar{Y}$, where $Y$ and $Y^{\prime}$ are strata.

Definition 3.3. A costratified Hilbert space relative to $N$ is a contravariant functor from $\mathcal{C}_{N}$ to the category of Hilbert spaces, with bounded linear maps as morphisms.

In more down to earth terms, a costratified Hilbert space relative to $N$ assigns a Hilbert space $\mathcal{C}_{Y}$ to each stratum $Y$, together with a bounded linear map $\mathcal{C}_{Y_{2}} \rightarrow \mathcal{C}_{Y_{1}}$ for each inclusion $Y_{1} \subseteq \overline{Y_{2}}$ such that, whenever $Y_{1} \subseteq \overline{Y_{2}}$ and $Y_{2} \subseteq \overline{Y_{3}}$, the composite of $\mathcal{C}_{Y_{3}} \rightarrow \mathcal{C}_{Y_{2}}$ with $\mathcal{C}_{Y_{2}} \rightarrow \mathcal{C}_{Y_{1}}$ coincides with the bounded linear map $\mathcal{C}_{Y_{3}} \rightarrow \mathcal{C}_{Y_{1}}$ associated with the inclusion $Y_{1} \subseteq \overline{Y_{3}}$.

To construct a costratified Hilbert space relative to the reduced phase space $\mathcal{P}$, we start with the Hilbert space $\mathcal{H} L^{2}\left(K^{\mathbb{C}}, \mathrm{e}^{-\kappa / \hbar} \eta \varepsilon\right)^{K}$ and single out subspaces $\mathcal{H}_{\tau, i}$ associated with the strata $\mathcal{P}_{\tau, i}$ as follows. The elements of $\mathcal{H} L^{2}\left(K^{\mathbb{C}}, \mathrm{e}^{-\kappa / \hbar} \eta \varepsilon\right)^{K}$ are ordinary 
functions on $K^{\mathbb{C}}$, not classes of functions as in the $L^{2}$-case. Therefore, being $K$-invariant, these functions define functions on $\mathcal{P}$. Thus, we associate with each stratum $\mathcal{P}_{\tau, i}$ of $\mathcal{P}$ the subspace

$$
\mathcal{V}_{\tau, i}=\left\{f \in \mathcal{H} L^{2}\left(K^{\mathbb{C}}, \mathrm{e}^{-\kappa / \hbar} \eta \varepsilon\right)^{K} ;\left.f\right|_{\mathcal{P}_{\tau, i}}=0\right\}
$$

of $\mathcal{H} L^{2}\left(K^{\mathbb{C}}, \mathrm{e}^{-\kappa / \hbar} \eta \varepsilon\right)^{K}$ which consists of the functions that vanish on $\mathcal{P}_{\tau, i}$. We then define the Hilbert space $\mathcal{H}_{\tau, i}$ associated with $\mathcal{P}_{\tau, i}$ to be the orthogonal complement of $\mathcal{V}_{\tau, i}$ in $\mathcal{H} L^{2}\left(K^{\mathbb{C}}, \mathrm{e}^{-\kappa / \hbar} \eta \varepsilon\right)^{K}$, so that $\mathcal{H} L^{2}\left(K^{\mathbb{C}}, \mathrm{e}^{-\kappa / \hbar} \eta \varepsilon\right)^{K}=\mathcal{V}_{\tau, i} \oplus \mathcal{H}_{\tau, i}$.

By construction, if $\mathcal{P}_{\tau_{1}, i_{1}} \subseteq \overline{\mathcal{P}_{\tau_{2}, i_{2}}}$ then $\mathcal{V}_{\tau_{2}, i_{2}} \subseteq \mathcal{V}_{\tau_{1}, i_{1}}$ and, therefore, $\mathcal{H}_{\tau_{1}, i_{1}} \subseteq \mathcal{H}_{\tau_{2}, i_{2}}$. Let $\Pi_{\tau_{2}, i_{2} ; \tau_{1}, i_{1}}: \mathcal{H}_{\tau_{2}, i_{2}} \rightarrow \mathcal{H}_{\tau_{1}, i_{1}}$ denote the orthogonal projection. The resulting system $\left\{\mathcal{H}_{\tau, i}\right\}$, together with the orthogonal projections $\Pi_{\tau_{2}, i_{2} ; \tau_{1}, i_{1}}: \mathcal{H}_{\tau_{2}, i_{2}} \rightarrow \mathcal{H}_{\tau_{1}, i_{1}}$ whenever $\mathcal{P}_{\tau_{1}, i_{1}} \subseteq \overline{\mathcal{P}_{\tau_{2}, i_{2}}}$, is the costratified Hilbert space relative to $\mathcal{P}$ we are looking for. When $\tau$ is the principal orbit type, $\mathcal{H}_{\tau}$ plainly coincides with the total Hilbert space $\mathcal{H} L^{2}\left(K^{\mathbb{C}}, \mathrm{e}^{-\kappa / \hbar} \eta \varepsilon\right)^{K}$.

While being defined in the holomorphic representation, the costratified Hilbert space structure may be transferred to the Schrödinger representation. In Sect. 4 we shall determine the costratified Hilbert space structure explicitly for the case $K=\mathrm{SU}(2)$.

3.4. Observables. The prequantization procedure assigns to a classical observable $f \in C^{\infty}\left(\mathrm{T}^{*} K\right)$ the operator $\hat{f}$ on the prequantum Hilbert space $L^{2}\left(\mathrm{~T}^{*} K, \varepsilon\right)$ given by

$$
\hat{f}=\mathrm{i} \hbar X_{f}+f-\frac{1}{\hbar} \theta\left(X_{f}\right)
$$

here $\theta$ is the symplectic potential (2.2), so that $-d \theta$ coincides with the cotangent bundle symplectic structure $\omega$ on $\mathrm{T}^{*} K$, and $X_{f}$ denotes the Hamiltonian vector field associated with $f$, determined by the identity

$$
\omega\left(X_{f}, \cdot\right)=d f
$$

in accordance with Hamilton's equations. The formula (3.13) is essentially the same as that given as (8.2.2) in [56], save that the Hamiltonian vector field $X_{f}$ and the symplectic potential $\theta$ are the negatives of the corresponding objects in [56]. Let $\{\cdot, \cdot\}$ be the Poisson structure on $C^{\infty}\left(\mathrm{T}^{*} K\right)$ associated with the cotangent bundle symplectic structure $\omega$; this Poisson structure is given by (2.3). Then $\hbar\{\cdot, \cdot\}$ is the Poisson structure on $C^{\infty}\left(\mathrm{T}^{*} K\right)$ associated with the symplectic structure $\frac{\omega}{\hbar}$. The formula (3.13) yields a representation of the Lie algebra underlying the Poisson algebra $\left(C^{\infty}\left(\mathrm{T}^{*} K\right), \hbar\{\cdot, \cdot\}\right)$ which satisfies the Dirac conditions. This representation is not irreducible and, to arrive at an irreducible representation of at least a certain subalgebra, the standard procedure is to introduce a polarization. Observables in this subalgebra are then referred to as being quantizable in the polarization under discussion.

In our situation, in the Kähler polarization, only the constants are quantizable. In the Schrödinger polarization, the topological obstruction to the existence of a half-form bundle vanishes for trivial reasons and, with the half-form correction incorporated, the relevant subalgebra of $C^{\infty}\left(\mathrm{T}^{*} K\right)$ contains the functions which restrict to polynomials of at most second order on the fibres of $\mathrm{T}^{*} K$, i. e., which are at most quadratic in the 
generalized momenta. Thus, it contains the (classical) Hamiltonian (2.5) of our model. The associated quantum observable, i. e., the (quantum) Hamiltonian, is given by

$$
H=-\frac{\hbar^{2}}{2} \Delta_{K}+\frac{v}{2}\left(3-\operatorname{Re} \chi_{\lambda_{1}}\right),
$$

where $\lambda_{1}$ denotes the highest weight of the defining representation of $K$. The operator $\Delta_{K}$ arises from the non-positive Laplace-Beltrami operator $\tilde{\Delta}_{K}$ associated with the bi-invariant Riemannian metric on $K$ as follows: The operator $\tilde{\Delta}_{K}$ is essentially selfadjoint on $C^{\infty}(K)$ and has a unique extension $\Delta_{K}$ to an (unbounded) self-adjoint operator on $L^{2}(K, d x)$. The spectrum being discrete, the domain of this extension is the space of functions of the form $f=\sum_{n} \alpha_{n} \varphi_{n}$ such that $\sum_{n}\left|\alpha_{n}\right|^{2} \lambda_{n}^{2}<\infty$, where the $\varphi_{n}$ 's range over the eigenfunctions and the $\lambda_{n}$ 's over the eigenvalues of $\tilde{\Delta}_{K}$.

Since the metric is bi-invariant, so is the operator $\Delta_{K}$, whence this operator restricts to a self-adjoint operator on the subspace $L^{2}(K, \mathrm{~d} x)^{K}$ which we continue to denote by $\Delta_{K}$. A core for this operator, and hence for the Hamiltonian $H$, is given by $C^{\infty}(K)^{K}$.

By means of the unitary transform (3.10) we now transfer the Hamiltonian and, in particular, the operator $\Delta_{K}$ to the holomorphic representation, i. e., to self-adjoint operators on $\mathcal{H} L^{2}\left(K^{\mathbb{C}}, \mathrm{e}^{-\kappa / \hbar} \eta \varepsilon\right)^{K}$. Concerning $\Delta_{K}$, we may alternatively view $\tilde{\Delta}_{K}$ as a differential operator on $K^{\mathbb{C}}$ via the embedding of $\mathfrak{k}$ into $\mathfrak{k}^{\mathbb{C}}$, extend it to a self-adjoint operator on $\mathcal{H} L^{2}\left(K^{\mathbb{C}}, \mathrm{e}^{-\kappa / \hbar} \eta \varepsilon\right)$, and take the restriction to the subspace $\mathcal{H} L^{2}\left(K^{\mathbb{C}}, \mathrm{e}^{-\kappa / \hbar} \eta \varepsilon\right)^{K}$.

Next, we determine the eigenvalues and the eigenfunctions of $\Delta_{K}$. The operator $\tilde{\Delta}_{K}$ is known to coincide with the Casimir operator on $K$ associated with the bi-invariant Riemannian metric, see [53] (A 1.2). That is to say, after a choice $X_{1}, \ldots, X_{m}$ of orthonormal basis of $\mathfrak{k}$ has been made,

$$
\tilde{\Delta}_{K}=X_{1}^{2}+\cdots+X_{m}^{2}
$$

in the universal enveloping algebra $U(\mathfrak{k})$ of $\mathfrak{k}$, cf. e. g. [48] (p. 591). Since $\tilde{\Delta}_{K}$ is bi-invariant, by Schur's lemma, each isotypical $(K \times K)$-summand $L^{2}(K, d x)_{\lambda}$ of $L^{2}(K, d x)$ in the Peter-Weyl decomposition is an eigenspace, and the representative functions are eigenfunctions for $\tilde{\Delta}_{K}$. The eigenvalue of $\tilde{\Delta}_{K}$ corresponding to the highest weight $\lambda$ is known to be given explicitly by $-\varepsilon_{\lambda}$, where

$$
\varepsilon_{\lambda}=\left(|\lambda+\rho|^{2}-|\rho|^{2}\right)
$$

cf. e. g. [27] (Chap. V.1 (16)). The sign is chosen in such a way that the $\varepsilon_{\lambda}$ can be interpreted as energy values. Hence, in particular, each character $\chi_{\lambda}$ is an eigenfunction of $\Delta_{K}$ associated with the eigenvalue $-\varepsilon_{\lambda}$. Consequently, $\Delta_{K}$ being viewed as an operator on the abstract Hilbert space $\mathcal{H}$, the vectors $|\lambda\rangle \in \mathcal{H}$ form an orthonormal eigenbasis of $\mathcal{H}$. In view of an observation spelled out above, the domain of $\Delta_{K}$ is explicitly given by

$$
\left\{\sum_{\lambda} \alpha_{\lambda}|\lambda\rangle \in \mathcal{H}: \sum_{\lambda}\left|\alpha_{\lambda}\right|^{2} \varepsilon_{\lambda}^{2}<\infty\right\}
$$

\section{The Costratified Hilbert Space for SU(2)}

4.1. Group theoretical data. The (real) root system of $\mathfrak{k}=\operatorname{su}(2)$ consists of the two roots $\alpha$ and $-\alpha$, given by

$$
\alpha(Y)=2 y, \quad Y \in \mathfrak{t}
$$


where $Y=\operatorname{diag}(\mathrm{i} y,-\mathrm{i} y), y \in \mathbb{R}$. Then $\varrho=\frac{1}{2} \alpha$. We label the irreducible representations by non-negative integers $n$ (twice the spin). The corresponding highest weights $\lambda_{n}$ are given by $\lambda_{n}=\frac{n}{2} \alpha$. On $T \times \mathfrak{t} \cong T^{\mathbb{C}}$, the corresponding complex characters $\chi_{n}^{\mathbb{C}}$ of $K^{\mathbb{C}}=\operatorname{SL}(2, \mathbb{C})$ are given by

$$
\chi_{n}^{\mathbb{C}}(t)=z^{n}+z^{n-2}+\cdots+z^{-n}, \quad t \in T^{\mathbb{C}},
$$

where $t=\operatorname{diag}\left(z, z^{-1}\right), z \in \mathbb{C}^{*}$. Restriction to $K$ yields the real characters which, on $T$, can be written as

$$
\chi_{n}(t)=\frac{\sin ((n+1) x)}{\sin (x)}, \quad t \in T,
$$

where $t=\operatorname{diag}\left(\mathrm{e}^{\mathrm{i} x}, \mathrm{e}^{-\mathrm{i} x}\right), x \in \mathbb{R}$. Any invariant inner product $\langle\cdot, \cdot\rangle$ on $\mathfrak{k}=\operatorname{su}(2)$ is proportional to the (negative definite) trace form. Hence, given $\langle\cdot, \cdot\rangle$, we can define a positive number $\beta$ by

$$
\left\langle Y_{1}, Y_{2}\right\rangle=-\frac{1}{2 \beta^{2}} \operatorname{tr}\left(Y_{1} Y_{2}\right), Y_{1}, Y_{2} \in \mathfrak{k}
$$

For the Killing form, $\beta=\frac{1}{\sqrt{8}}$. Relative to the given invariant inner product on $\mathfrak{k}$, the two roots $\alpha$ and $-\alpha$ have norm $|\alpha|^{2}=4 \beta^{2}$. Hence $|\varrho|^{2}=\beta^{2}$ and $\left|\lambda_{n}+\varrho\right|^{2}=\beta^{2}(n+1)^{2}$, whence according to (3.9) and (3.15)

$$
\varepsilon_{n}=\beta^{2} n(n+2), \quad C_{n}=(\hbar \pi)^{3 / 2} \mathrm{e}^{\hbar \beta^{2}(n+1)^{2}} .
$$

4.2. The costratified Hilbert space structure. According to Sect. 3, the appropriate Hilbert space for the holomorphic representation is the Hilbert space $\mathcal{H} L^{2}\left(K^{\mathbb{C}}, \mathrm{e}^{-\kappa / \hbar} \eta \varepsilon\right)^{K}$ or, equivalently, $\mathcal{H} L^{2}\left(T^{\mathbb{C}}, \mathrm{e}^{-\kappa / \hbar} \gamma \varepsilon_{T}\right)^{W}$. The Hilbert space for the Schrödinger representation is the space $L^{2}(K, \mathrm{~d} x)^{K}$ or, equivalently, $L^{2}(T, v \mathrm{~d} t)^{W}$. There is no need to spell out the functions $\kappa, \eta, \gamma$ or $v$ here, because we can work entirely in the basis given by the characters. For $n \geq 0$, let $|n\rangle:=\left|\lambda_{n}\right\rangle$; then $\{|n\rangle: n=0,1,2, \ldots\}$ is an orthonormal basis of $\mathcal{H}$, and we can pass to the holomorphic and to the Schrödinger representation by replacing each $|n\rangle$ with the corresponding (normalized) character.

We now determine the costratified Hilbert space structure constituents $\mathcal{H}_{ \pm}$and $\mathcal{H}_{1}$ associated with the strata $\mathcal{P}_{ \pm}$and $\mathcal{P}_{1}$ of $\mathcal{P}$ and the subspace $\mathcal{H}_{0}$ associated with the orbit type subset $\mathcal{P}_{0}$. Recall the notation and the description of these strata from Subsect. 2.3. As $\mathcal{P}_{1}$ is the top stratum, $\mathcal{H}_{1}=\mathcal{H}$. To describe the subspaces $\mathcal{H}_{ \pm}$and $\mathcal{H}_{0}$, we pass to the holomorphic representation.

Lemma 4.1. The systems (4.4), (4.5), and (4.6) below constitute bases of, respectively, the subspaces $\mathcal{V}_{+}, \mathcal{V}_{-}, \mathcal{V}_{0}$ of $\mathcal{H}$ corresponding to, respectively, the strata $\mathcal{P}_{+}, \mathcal{P}_{-}$and $\mathcal{P}_{0}$ :

$$
\begin{aligned}
& \chi_{n}^{\mathbb{C}}-(n+1) \chi_{0}^{\mathbb{C}}, \quad n=1,2,3, \ldots, \\
& \chi_{n}^{\mathbb{C}}+(-1)^{n} \frac{n+1}{2} \chi_{1}^{\mathbb{C}}, \quad n=0,2,3, \ldots, \\
& \chi_{2 k}^{\mathbb{C}}-(2 k+1) \chi_{0}^{\mathbb{C}}, \quad \chi_{2 k+1}^{\mathbb{C}}-(k+1) \chi_{1}^{\mathbb{C}}, \quad k=1,2,3, \ldots
\end{aligned}
$$


Proof. We view the elements of $\mathcal{H}$ as functions on $T^{\mathbb{C}}$ rather than on $K^{\mathbb{C}}$. Via the polar decomposition map $T \times \mathfrak{t} \rightarrow T^{\mathbb{C}}$, the points $( \pm \mathbb{1}, 0)$ are mapped to $\{ \pm \mathbb{1}\}$. Hence, $\mathcal{V}_{+}$, $\mathcal{V}_{-}$and $\mathcal{V}_{0}$ consist of the functions $\psi \in \mathcal{H}$ that satisfy, respectively,

$$
\psi(\mathbb{1})=0, \quad \psi(-\mathbb{1})=0, \quad \psi( \pm \mathbb{1})=0 .
$$

Due to $\chi_{n}^{\mathbb{C}}( \pm \mathbb{1})=( \pm 1)^{n}(n+1)$, we have

$$
\chi_{2 k}^{\mathbb{C}}( \pm \mathbb{1})=2 k+1=(2 k+1) \chi_{0}^{\mathbb{C}}( \pm \mathbb{1})
$$

and

$$
\chi_{2 k+1}^{\mathbb{C}}( \pm \mathbb{1})= \pm(2 k+2)=(k+1) \chi_{1}^{\mathbb{C}}( \pm \mathbb{1})
$$

Hence, all the functions given in (4.4)-(4.6) satisfy the corresponding condition in (4.7). Conversely, given $\psi \in \mathcal{V}_{+}$, expanding it in the basis of $\mathcal{H}$ given by the elements in (4.4) together with $\chi_{0}^{\mathbb{C}}$ we see that the vanishing of $\psi(\mathbb{1})$ implies that the coefficient of $\chi_{0}^{\mathbb{C}}$ is zero. The reasoning for $\mathcal{V}_{-}$and $\mathcal{V}_{0}$ is analogous. Finally, linear independence of the systems (4.4)-(4.6) is obvious.

We express the bases (4.4)-(4.6), up to a common factor $(\hbar \pi)^{3 / 4}$, in terms of $|n\rangle$ :

$$
\begin{aligned}
& \mathrm{e}^{\hbar \beta^{2}(n+1)^{2} / 2}|n\rangle-(n+1) \mathrm{e}^{\hbar \beta^{2} / 2}|0\rangle, \quad n=1,2,3, \ldots, \\
& \mathrm{e}^{\hbar \beta^{2}(n+1)^{2} / 2}|n\rangle-\frac{n+1}{2} \mathrm{e}^{2 \hbar \beta^{2}}|1\rangle, \quad n=0,2,3, \ldots, \\
& \mathrm{e}^{\hbar \beta^{2}(2 k+1)^{2} / 2}|2 k\rangle-(2 k+1) \mathrm{e}^{\hbar \beta^{2} / 2}|0\rangle, \quad k=1,2,3, \ldots, \\
& \mathrm{e}^{2 \hbar \beta^{2}(k+1)^{2}}|2 k+1\rangle-(k+1) \mathrm{e}^{2 \hbar \beta^{2}}|1\rangle, \quad k=1,2,3, \ldots
\end{aligned}
$$

Proposition 4.2. The subspaces $\mathcal{H}_{+}$and $\mathcal{H}_{-}$have dimension 1 . They are spanned by the normalized vectors

$$
\begin{aligned}
\psi_{+} & :=\frac{1}{N} \sum_{n=0}^{\infty}(n+1) \mathrm{e}^{-\hbar \beta^{2}(n+1)^{2} / 2}|n\rangle, \\
\psi_{-} & :=\frac{1}{N} \sum_{n=0}^{\infty}(-1)^{n}(n+1) \mathrm{e}^{-\hbar \beta^{2}(n+1)^{2} / 2}|n\rangle,
\end{aligned}
$$

respectively. The subspace $\mathcal{H}_{0}$ has dimension 2 . It is spanned by the orthonormal basis

$$
\psi_{\mathrm{g}}:=\frac{1}{N_{\mathrm{g}}} \sum_{n \text { even }}(n+1) \mathrm{e}^{-\hbar \beta^{2}(n+1)^{2} / 2}|n\rangle, \quad \psi_{\mathrm{u}}:=\frac{1}{N_{\mathrm{u}}} \sum_{n \text { odd }}(n+1) \mathrm{e}^{-\hbar \beta^{2}(n+1)^{2} / 2}|n\rangle,
$$

where the sum over the even $n$ includes $n=0$. The normalization factors are

$$
N^{2}=\sum_{n=1}^{\infty} n^{2} \mathrm{e}^{-\hbar \beta^{2} n^{2}}, \quad N_{\mathrm{g}}^{2}=\sum_{n \text { odd }} n^{2} \mathrm{e}^{-\hbar \beta^{2} n^{2}}, \quad N_{\mathrm{u}}^{2}=\sum_{n \text { even }} n^{2} \mathrm{e}^{-\hbar \beta^{2} n^{2}} .
$$


Proof. The sums in (4.11), (4.12) and (4.13) converge, their limits are normalized, and $\psi_{\mathrm{g}}$ and $\psi_{\mathrm{u}}$ are mutually orthogonal. The vector $\psi_{+}$together with the system (4.8), $\psi_{-}$ together with the system (4.9), and $\psi_{\mathrm{g}}, \psi_{\mathrm{u}}$ together with the system (4.10) provide bases of $\mathcal{H}$. Finally, it is straightforward to check that $\psi_{+}, \psi_{-}$and $\psi_{\mathrm{g}}, \psi_{\mathrm{u}}$ are orthogonal to the corresponding system in (4.8)-(4.10).

Proposition 4.2 implies that, in Dirac notation, for $i=0, \pm$, the orthogonal projections $\Pi_{i} \equiv \Pi_{1 i}: \mathcal{H}_{1} \rightarrow \mathcal{H}_{i}$ are given by

$$
\Pi_{ \pm}=\left|\psi_{ \pm}\right\rangle\left\langle\psi_{ \pm}\left|, \quad \Pi_{0}=\right| \psi_{\mathrm{g}}\right\rangle\left\langle\psi_{\mathrm{g}}|+| \psi_{\mathrm{u}}\right\rangle\left\langle\psi_{\mathrm{u}}\right| .
$$

The normalization factors $N, N_{\mathrm{g}}$ and $N_{\mathrm{u}}$ can be expressed in terms of the $\theta$-constant $\theta_{3}$ with 'nome' $Q$ as

$$
\theta_{3}(Q)=\sum_{k=-\infty}^{\infty} Q^{k^{2}}
$$

For example,

$$
N^{2}=-\frac{\partial}{\partial\left(\hbar \beta^{2}\right)} \sum_{n=1}^{\infty} \mathrm{e}^{-\hbar \beta^{2} n^{2}}=-\frac{1}{2} \frac{\partial}{\partial\left(\hbar \beta^{2}\right)} \theta_{3}\left(\mathrm{e}^{-\hbar \beta^{2}}\right)=\frac{1}{2} \mathrm{e}^{-\hbar \beta^{2}} \theta_{3}^{\prime}\left(\mathrm{e}^{-\hbar \beta^{2}}\right) .
$$

Then

$$
N_{\mathrm{u}}^{2}=4 \mathrm{e}^{-4 \hbar \beta^{2}} \theta_{3}^{\prime}\left(\mathrm{e}^{-4 \hbar \beta^{2}}\right), \quad N_{\mathrm{g}}^{2}=N^{2}-N_{\mathrm{u}}^{2}
$$

Remark 4.3. Let $\rho_{\hbar}(a):=\mathrm{e}^{\hbar \beta^{2}} N \psi_{+}$. Using (4.11) and plugging in for $|n\rangle$ the real characters $\chi_{n}$ we see that $\rho_{\hbar}$ satisfies the heat equation $\frac{\mathrm{d}}{\mathrm{d} \hbar} \rho=\frac{1}{2} \Delta_{K} \rho_{\hbar}$ subject to the initial condition $\rho_{0}=\delta_{\mathbb{1}}$, i.e., $\rho_{t}$ is the heat kernel of $K$. The expansion of $\rho_{\hbar}$ obtained from (4.11) is the standard expansion of the heat kernel of a compact Lie group in terms of its characters [52, p. 38]. According to [21, §4, Prop. 1], the function $\rho_{\hbar}$ has an analytic continuation to $K^{\mathbb{C}}$. This analytic continuation does not consist in substitution of the character $\chi_{n}^{\mathbb{C}}$ for the character $\chi_{n}$ in the standard expansion; in particular, the resulting formal series does not converge in $\mathcal{H} L^{2}\left(K^{\mathbb{C}}, \mathrm{e}^{-\kappa / \hbar} \eta \varepsilon\right)^{K}$. Thus $\rho_{\hbar}$ defines the complex-valued functions

$$
\psi_{g}^{(\hbar)}(a)=\overline{\rho_{\hbar}\left(g a^{-1}\right)}, \quad a \in K,
$$

on $K$, parametrized by the members $g \in K^{\mathbb{C}}$. According to [21], these functions admit an interpretation as coherent states on $K$. Indeed, the functions $\psi_{ \pm}$and $\psi_{ \pm \mathbb{1}}^{(\hbar)}$ are related by the identity

$$
\psi_{ \pm}=\frac{\mathrm{e}^{-\hbar \beta^{2}}}{N} \psi_{ \pm \mathbb{1}}^{(\hbar)}
$$

i.e., up to a normalization factor, the states spanning the subspaces $\mathcal{H}_{ \pm}$are the coherent states labelled by the points of the corresponding strata. This observation is certainly not a coincidence; in fact, for physical reasons, the states which the functions $\psi_{ \pm}$represent should come down to coherent states because the (phase space) wave function orthogonal to all wave functions vanishing at a given point represents a state of optimal localization in phase space (i.e., minimal position-momentum uncertainty). This is exactly what is generally understood to be a coherent state. 


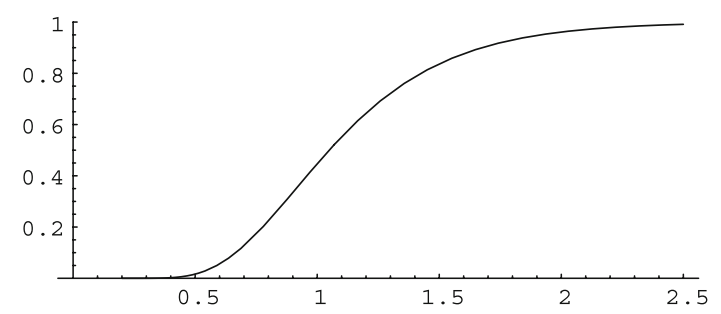

Fig. 2. Tunneling probability $\left|\left\langle\psi_{+}, \psi_{-}\right\rangle\right|^{2}$ as a function of $\hbar \beta^{2}$

4.3. Tunneling between strata. Consider the constituents $\mathcal{H}_{+}$and $\mathcal{H}_{-}$of the costratified Hilbert space $\mathcal{H}$ relative to the orbit type stratification of $\mathcal{P}$. A straightforward calculation yields

$$
\left\langle\psi_{+}, \psi_{-}\right\rangle=\frac{1}{N^{2}} \sum_{n=1}^{\infty}(-1)^{n+1} n^{2} \mathrm{e}^{-\hbar \beta^{2} n^{2}}=\frac{N_{\mathrm{g}}^{2}-N_{\mathrm{u}}^{2}}{N^{2}} .
$$

As in Subsect. 4.2 above, the scalar product can be expressed in terms of $\theta$-functions. Likewise, as in (4.16) for $N^{2}$, the alternating sum in the denominator can be rewritten as $-\mathrm{e}^{-\hbar \beta^{2}} \theta_{3}^{\prime}\left(-\mathrm{e}^{-\hbar \beta^{2}}\right)$. Together with (4.16) this yields

$$
\left\langle\psi_{+}, \psi_{-}\right\rangle=-\frac{\theta_{3}^{\prime}\left(-\mathrm{e}^{-\hbar \beta^{2}}\right)}{\theta_{3}^{\prime}\left(\mathrm{e}^{-\hbar \beta^{2}}\right)} .
$$

The absolute square $\left|\left\langle\psi_{+}, \psi_{-}\right\rangle\right|^{2}$ is the tunneling probability between the strata $\mathcal{P}_{+}$and $\mathcal{P}_{-}$, i. e., the probability for a state prepared at $\mathcal{P}_{+}$to be measured at $\mathcal{P}_{-}$and vice versa.

The numerical value of this quantity strongly depends on the combined constant $\hbar \beta^{2}$, see Fig. 2. For large values of $\hbar \beta^{2},\left|\left\langle\psi_{+}, \psi_{-}\right\rangle\right|^{2}$ is almost equal to 1 . This can also be read off from the expansions (4.11) and (4.12): the first coefficient that distinguishes between $\psi_{+}$and $\psi_{-}$is $2 e^{-4 \hbar \beta^{2}}$; for large $\hbar \beta^{2}$, this coefficient is much smaller than the leading coefficient $\mathrm{e}^{-\hbar \beta^{2}}$, so that $\psi_{+}$and $\psi_{-}$have a large overlap. In fact, in the limit $\hbar \beta^{2} \rightarrow \infty$ they become both equal to $|0\rangle$.

On the other hand, for $\hbar \beta^{2} \rightarrow 0$ we have $\left|\left\langle\psi_{+}, \psi_{-}\right\rangle\right|^{2} \rightarrow 0$. Thus, in the semiclassical limit, the tunneling probability vanishes.

Remark 4.4. Since the strata $\mathcal{P}_{+}$and $\mathcal{P}_{-}$together constitute the orbit type subset $\mathcal{P}_{0}$, a tunneling between them should not be visible in the costratification given by $\mathcal{H}_{0}$, that is, in the costratification relative to the coarser decomposition $\mathcal{P}=\mathcal{P}_{0} \cup \mathcal{P}_{1}$ by mere orbit types (and not by the connected components thereof). Indeed, we have

$$
\mathcal{H}_{0}=\mathcal{H}_{+} \oplus \mathcal{H}_{-},
$$

where the sum is direct but not orthogonal, and $\psi_{ \pm}$can be written as

$$
\psi_{ \pm}=\frac{N_{\mathrm{g}}}{N} \psi_{\mathrm{g}} \pm \frac{N_{\mathrm{u}}}{N} \psi_{\mathrm{u}}
$$

In other words, the subspaces $\mathcal{H}_{+}$and $\mathcal{H}_{-}$are swallowed by $\mathcal{H}_{0}$ and there is no way to reconstruct them from $\mathcal{H}_{0}$ alone. 
Remark 4.5. Expressing the scalar product in terms of the coherent states $\psi_{\mathbb{1}}^{(\hbar)}$ and $\psi_{-\mathbb{1}}^{(\hbar)}$, see Remark 4.3, we obtain the identity

$$
\left|\left\langle\psi_{+}, \psi_{-}\right\rangle\right|^{2}=\frac{\left|\left\langle\psi_{\mathbb{1}}^{(\hbar)}, \psi_{-\mathbb{1}}^{(\hbar)}\right\rangle\right|^{2}}{\left\|\psi_{\mathbb{1}}^{(\hbar)}\right\|^{2}\left\|\psi_{-\mathbb{1}}^{(\hbar)}\right\|^{2}}
$$

The quantity $\frac{\left|\left\langle\psi_{g}^{(\hbar)}, \psi_{h}^{(\hbar)}\right\rangle\right|^{2}}{\left\|\psi_{g}^{(\hbar)}\right\|^{2}\left\|\psi_{h}^{(\hbar)}\right\|^{2}}$ is known as the overlap of the coherent states $\psi_{g}^{(\hbar)}$ and $\psi_{h}^{(\hbar)}$; it was studied in more general situations in great detail in a series of papers by Thiemann and collaborators [54,55]. Among other things, they have shown that for $K=\mathrm{SU}(2)$ the overlap is related with the geodesic distance on $K^{\mathbb{C}}=\operatorname{SL}(2, \mathbb{C})$ and that, in general, for $g \neq h$ and $\hbar \rightarrow 0$, the overlap vanishes faster than any power of $\hbar$.

The scalar product $\left\langle\psi_{g}^{(\hbar)}, \psi_{h}^{(\hbar)}\right\rangle$, viewed as a function of $g$ and $h$, is known as the reproducing kernel associated with the family of coherent states $\psi_{g}^{(\hbar)}, g \in K^{\mathbb{C}}$. It can be expressed in terms of the heat kernel $\rho_{\hbar}$. For $\mathrm{SU}(2)$, this leads to Formula (4.18).

4.4. Adapted orthonormal bases. For $i= \pm$, 0 , we will now construct orthonormal bases of the subspaces $\mathcal{V}_{i}$ of $\mathcal{H}$. To this end, let

$$
\hat{\psi}_{ \pm}:=\frac{\left(1-\Pi_{\mp}\right) \psi_{ \pm}}{\left\|\left(1-\Pi_{\mp}\right) \psi_{ \pm}\right\|} .
$$

Then $\mathcal{V}_{ \pm}=\mathcal{V}_{0} \oplus \mathbb{C} \hat{\psi}_{\mp}$, the sum being orthogonal since $\hat{\psi}_{ \pm} \in \mathcal{H}_{0}$. Hence, it suffices to construct an orthonormal basis of $\mathcal{V}_{0}$. For that purpose, we orthonormalize the family (4.10). This can of course be done for the even and odd degree families separately.

Lemma 4.6. Let $\varphi_{n}, n=0,1,2, \ldots$ be an orthonormal basis of the Hilbert space $\mathcal{E}$ and let $f_{n}, n=0,1,2, \ldots$, be real numbers with $f_{0}=1$. Then orthonormalization of the system $\varphi_{n}-f_{n} \varphi_{0}, n=1,2,3, \ldots$, yields the system

$$
\tilde{\varphi}_{n}=\frac{F_{n-1}}{F_{n}} \varphi_{n}-\frac{f_{n}}{F_{n} F_{n-1}} \sum_{k=0}^{n-1} f_{k} \varphi_{k}, \quad n=1,2,3, \ldots
$$

where $F_{n}^{2}=\sum_{k=0}^{n} f_{k}^{2}$.

Proof. Straightforward calculation.

Let $\psi_{2 n}, n=1,2,3, \ldots$, denote the basis elements obtained by application of Lemma 4.6 to the even degree family of (4.10). Thus substituting $|2 k\rangle$ for $\varphi_{k}$ and $(2 k+1) \mathrm{e}^{-\hbar \varepsilon_{2 k} / 2}$ for $f_{k}$ in (4.20) yields $\psi_{2 n}$. Likewise, let $\psi_{2 n+1}, n=1,2,3, \ldots$, denote the basis elements obtained by applying the lemma to the odd degree family of (4.10), so that substituting $|2 k+1\rangle$ for $\varphi_{k}$ and $(k+1) \mathrm{e}^{-2 \hbar \varepsilon_{k}}$ for $f_{k}$ in (4.20) yields $\psi_{2 n+1}$.

The resulting vectors $\psi_{n}, n=2,3,4, \ldots$ form an orthonormal basis of $\mathcal{V}_{0}$. Adding $\hat{\psi}_{-}$, we obtain an orthonormal basis of $\mathcal{V}_{+}$. Adding $\hat{\psi}_{+}$, we obtain an orthonormal basis of $\mathcal{V}_{-}$. 
4.5. Representation in terms of $L^{2}[0, \pi]$. From now on we will work in the Schrödinger representation, i.e., we realize $\mathcal{H}$ as

$$
L^{2}(K)^{K} \cong L^{2}(T, v \mathrm{~d} t)^{W} .
$$

In order to produce plots of wave functions $\psi \in \mathcal{H}$ we choose a suitable parameterization of $\mathcal{X}$ and represent the elements of $\mathcal{H}$ by ordinary $L^{2}$-integrable functions on the parameter space. This representation will also be used in the discussion of the stationary Schrödinger equation of our model in Sect. 5. A suitable parameterization of $\mathcal{X}$ can be obtained as follows. We parameterize $T$ by

$$
\operatorname{diag}\left(\mathrm{e}^{\mathrm{i} x}, \mathrm{e}^{-\mathrm{i} x}\right), \quad x \in[-\pi, \pi] .
$$

Since the nontrivial element of $W$ acts by reflection $x \mapsto-x$, restriction of the parameter $x$ to the interval $[0, \pi]$ yields a (bijective) parameterization of $\mathcal{X}$, where $\mathcal{X}_{+}$corresponds to $x=0$ and $\mathcal{X}_{-}$to $x=\pi$.

In the parameterization (4.21), the measure $v \mathrm{~d} t$ on $T$ is given by

$$
v \mathrm{~d} t=\frac{\operatorname{vol}(K)}{\pi} \sin ^{2}(x) \mathrm{d} x .
$$

Hence, the assignment to $\psi \in C^{\infty}(T)^{W}$ of the function $x \mapsto \psi\left(\operatorname{diag}\left(\mathrm{e}^{\mathrm{i} x}, \mathrm{e}^{-\mathrm{i} x}\right)\right)$, $x \in[0, \pi]$, defines a Hilbert space isomorphism

$$
\Gamma_{1}: \mathcal{H} \rightarrow L^{2}\left([0, \pi], \sin ^{2}(x) \mathrm{d} x\right) .
$$

Furthermore, multiplication by $\sqrt{2} \sin x$ defines a Hilbert space isomorphism

$$
\Gamma_{2}: L^{2}\left([0, \pi], \sin ^{2}(x) \mathrm{d} x\right) \rightarrow L^{2}[0, \pi] .
$$

Here the scalar products in $L^{2}\left([0, \pi], \sin ^{2}(x) \mathrm{d} x\right)$ and $L^{2}[0, \pi]$ are normalized so that the constant function with value 1 has norm 1 .

The composite isomorphism $\Gamma=\Gamma_{2} \circ \Gamma_{1}$ identifies $\mathcal{H}$ with the space $L^{2}[0, \pi]$ of ordinary square-integrable functions on $[0, \pi]$. Plotting the function $\Gamma \psi$ rather than $\psi$ has the advantage that one can read off directly from the graph the corresponding probability density with respect to Lebesgue measure on the parameter space $[0, \pi]$.

Plots of $\Gamma \psi_{i}, i= \pm, \mathrm{g}, \mathrm{u}$, are shown in Fig. 3 for $\hbar \beta^{2}=\frac{1}{2}, \frac{1}{8}, \frac{1}{32}, \frac{1}{128}$. We remark that the value $\hbar \beta^{2}=1 / 8$ appears when we choose $\hbar=1$ and the negative of the Killing form as the invariant scalar product on $\mathfrak{k}$. Moreover, according to (4.2),

$$
\left(\Gamma \chi_{n}\right)(x)=\sqrt{2} \sin ((n+1) x),
$$

hence the expansions (4.11)-(4.13) boil down to ordinary Fourier expansions of the functions $\Gamma \psi_{i}, i= \pm, \mathrm{g}, \mathrm{u}$.
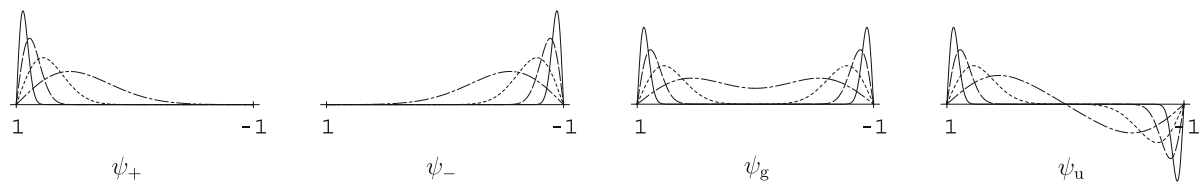

Fig. 3. Plots of images of the wave functions $\psi_{i}, i= \pm$, g, u under $\Gamma$, for $\hbar \beta^{2}=1 / 128$ (continuous line), 1/32 (long dash), 1/8 (short dash), 1/2 (alternating short-long dash) 


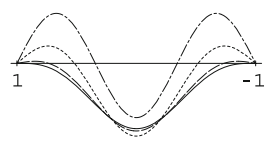

$\psi_{2}$

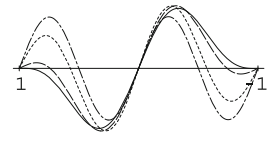

$\psi_{3}$

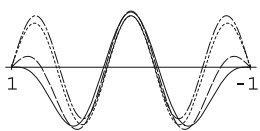

$\psi_{4}$

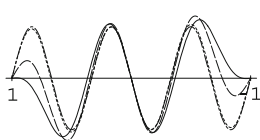

$\psi_{5}$
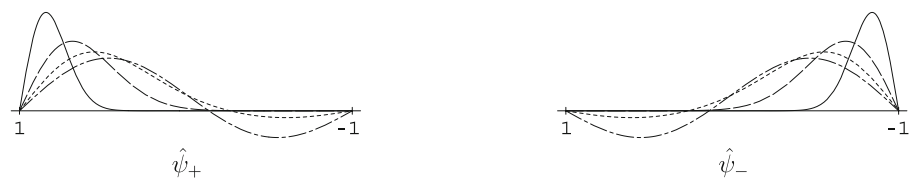

Fig. 4. Plots of the images of the wave functions $\psi_{2}, \ldots, \psi_{4}$ and $\hat{\psi}_{ \pm}$, under $\Gamma$, for $\hbar \beta^{2}=\frac{1}{16}$ (continuous line), $\frac{1}{4}$ (long dash), $\frac{1}{2}$ (short dash), 1 (alternating short-long dash)

Plots of $\psi_{2}, \ldots, \psi_{5}$ and $\hat{\psi}_{ \pm}$are shown in Fig. 4 for $\hbar \beta^{2}=1, \frac{1}{2}, \frac{1}{4}, \frac{1}{16}$. For $\hbar \beta^{2} \rightarrow 0$, the outer nodes of the $\Gamma \psi_{n}$ run into the points $\mathcal{X}_{ \pm}$and thus decrease the number of nodes to $n-2$. Moreover, since for decreasing value of $\hbar \beta^{2}$ the overlap $\left\langle\psi_{+}, \psi_{-}\right\rangle$decreases, the functions $\hat{\psi}_{ \pm}$converge to $\psi_{ \pm}$.

\section{Energy Eigenvalues and Eigenstates for SU(2)}

We now determine the energy eigenvalues and the corresponding eigenfunctions of our model for $K=\mathrm{SU}(2)$. We start with a general discussion of the Hamiltonian.

5.1. The Hamiltonian. In the Schrödinger representation, the Hamiltonian is given by (3.14). It is a self-adjoint operator on the Hilbert space $L^{2}(K, \mathrm{~d} x)^{K} \equiv L^{2}(T, v \mathrm{~d} t)^{W}$. For domain issues it suffices to consider the kinetic part, i. e., the Laplacian $\Delta_{K}$. As a core we may take $C^{\infty}(K)^{K} \equiv C^{\infty}(T)^{W}$. According to (3.16), the full domain is

$$
\left\{\sum_{n=0}^{\infty} \alpha_{n} \chi_{n} \in L^{2}(K, \mathrm{~d} x)^{K}: \sum_{n=0}^{\infty}\left|\alpha_{n}\right|^{2} n^{2}(n+2)^{2}<\infty\right\} .
$$

The isomorphisms $\Gamma_{1}$ and $\Gamma_{2}$, see (4.22) and (4.23), carry $\Delta_{K}$ and $H$ to the selfadjoint operators

$$
\begin{aligned}
& \Delta_{1}=\Gamma_{1} \circ \Delta_{K} \circ \Gamma_{1}^{-1}, \quad \Delta_{2}=\Gamma_{2} \circ \Delta_{1} \circ \Gamma_{2}^{-1} \equiv \Gamma \circ \Delta_{K} \circ \Gamma^{-1} \\
& H_{1}=\Gamma_{1} \circ H \circ \Gamma_{1}^{-1}, \quad H_{2}=\Gamma_{2} \circ H_{1} \circ \Gamma_{2}^{-1} \equiv \Gamma \circ H \circ \Gamma^{-1}
\end{aligned}
$$

on the Hilbert spaces $L^{2}\left([0, \pi], \sin ^{2} x \mathrm{~d} x\right)$ and $L^{2}[0, \pi]$, respectively. Then

$$
H_{i}=-\frac{\hbar^{2}}{2} \Delta_{i}+\frac{v}{2}(3-2 \cos x), \quad i=1,2,
$$

where, formally,

$$
\Delta_{1}=\beta^{2}\left(\frac{1}{\sin (x)} \frac{\mathrm{d}^{2}}{\mathrm{~d} x^{2}} \sin (x)+1\right), \quad \Delta_{2}=\beta^{2}\left(\frac{\mathrm{d}^{2}}{\mathrm{~d} x^{2}}+1\right) .
$$

The formula for $\Delta_{1}$ follows from the general formula for the radial part of the Laplacian on a compact group, see [27, §II.3.4], or by explicitly applying this operator to the functions $\Gamma_{1} \chi_{n}$. 
Let $C^{\infty}[0, \pi]$ denote the space of Whitney smooth complex functions on the closed interval $[0, \pi]$. These are the smooth functions on the open interval $] 0, \pi[$ that can be extended to smooth functions on $\mathbb{R}$. In particular, the elements of $C^{\infty}[0, \pi]$ have well-defined derivatives of arbitrary order at 0 and at $\pi$.

Proposition 5.1. A core for $\Delta_{1}$ is given by $D_{1}=\left\{\psi \in C^{\infty}[0, \pi]: \psi^{\prime}(0)=\psi^{\prime}(\pi)=0\right\}$. A core for $\Delta_{2}$ is given by $D_{2}=\left\{\psi \in C^{\infty}[0, \pi]: \psi(0)=\psi(\pi)=0\right\}$.

Proof. First, consider $\Delta_{1}$. We have to show that

(a) $\Gamma_{1}\left(C^{\infty}(K)^{K}\right) \subseteq D_{1}$,

(b) $\Delta_{1}\left(D_{1}\right) \subseteq L^{2}\left([0, \pi], \sin ^{2} x \mathrm{~d} x\right)$,

(c) $\Delta_{1}$ is symmetric on $D_{1}$.

We may replace $\Delta_{1}$ with the operator $\tilde{\Delta}_{1}=\frac{1}{\sin x} \frac{\mathrm{d}^{2}}{\mathrm{~d} x^{2}} \sin x$. Concerning (a), we observe that the algebra of real invariant polynomials on $K=\mathrm{SU}(2)$ is generated by the trace monomial $\rho(a)=\frac{1}{2} \operatorname{tr}(a)$. A theorem in [50] states that $C^{\infty}(K)^{K}=\rho^{*} C^{\infty}(\mathbb{R})$. Hence, for given $\psi \in C^{\infty}(K)^{K}$ there exists $\varphi \in C^{\infty}(\mathbb{R})$ such that $\psi=\varphi \circ \rho$. Then $\left(\Gamma_{1} \psi\right)(x)=$ $\varphi(\cos x)$ and thus $\left(\Gamma_{1} \psi\right)^{\prime}(x)=-h^{\prime}(\cos x) \sin x$ vanishes for $x=0, \pi$.

To check (b), let $\psi \in D_{1}$. It suffices to show that the values of the function $\left(\tilde{\Delta}_{1} \psi\right)(x)$, $0<x<\pi$, converge for $x \rightarrow 0$ and $x \rightarrow \pi$. Since $\psi(0)$ and $\psi^{\prime \prime}(0)$ exist,

$$
\lim _{x \rightarrow 0}\left(\tilde{\Delta}_{1} \psi(x)\right)=\psi^{\prime \prime}(0)-\psi(0)+2 \lim _{x \rightarrow 0} \frac{\cos x \psi^{\prime}(x)}{\sin x} .
$$

Since $\lim _{x \rightarrow 0} \psi^{\prime}(x)=0$, we can apply the rule of Bernoulli and de l'Hospital. This yields

$$
\lim _{x \rightarrow 0}\left(\tilde{\Delta}_{1} \psi(x)\right)=3 \psi^{\prime \prime}(0)-\psi(0) .
$$

The reasoning for $x \rightarrow \pi$ is analogous.

To prove (c), let $\psi, \varphi \in D_{1}$. Then, omitting the normalization factor $2 / \pi$, we find

$$
\begin{aligned}
\int_{0}^{\pi} \overline{\psi(x)}\left(\tilde{\Delta}_{1} \varphi\right)(x) \sin ^{2} x \mathrm{~d} x= & \int_{0}^{\pi} \overline{\left(\tilde{\Delta}_{1} \psi\right)(x)} \varphi(x) \sin ^{2} x \mathrm{~d} x \\
& \quad+\left.\sin x \psi(x)(\sin x \varphi(x))^{\prime}\right|_{0} ^{\pi}-\left.\sin x \varphi(x)(\sin x \psi(x))^{\prime}\right|_{0} ^{\pi} .
\end{aligned}
$$

The boundary terms vanish because $\psi(x), \psi^{\prime}(x), \varphi(x)$ and $\varphi^{\prime}(x)$ exist for $x=0$ and $x=\pi$.

Next, consider $\Delta_{2}$. We have to check conditions (a)-(c) with the subscript 1 replaced with the subscript 2 , with $L^{2}\left([0, \pi], \sin ^{2} x \mathrm{~d} x\right)$ instead of $L^{2}[0, \pi]$, and with $C^{\infty}(K)^{K}$ instead of $D_{1}$. Conditions (a) and (b) are trivially satisfied and the verification of (c) is analogous to that for $\Delta_{1}$.

Remark 5.1. The operator $\Delta_{1}$ is discussed in [57, §4] as a specific example of a reduced Laplacian obtained by Rieffel induction. There, the same core is isolated. In our concrete situation the proof is much simpler than in the general setting of [57], though.

In view of the proposition, we will now discuss two items. First, we will relate our system with two standard elementary quantum mechanical systems. Thereafter, we will make a remark on the extension problem of the Hamiltonian in a 'naive' quantizationafter-reduction procedure. 
The proposition implies that, for $v=0$, the Hilbert space isomorphism $\Gamma$ maps our original system to that of a particle of mass $m=\frac{1}{2 \beta^{2}}$ moving in a one-dimensional square potential well of width $\pi$ with infinitely high walls. Inside the well the energy is shifted by $\beta^{2}=\frac{1}{2 m}$. For $\nu \neq 0$, the potential inside the square well is further modified by a cosine. This corresponds to a planar pendulum that is bound to move in one half of the circle only and is reflected elastically at the two equilibria. It would be interesting to clarify the relevance of the subspaces $\mathcal{H}_{ \pm}$in both these systems.

The relationship with the pendulum is in fact more intimate: Multiplication by the function $\sqrt{2} \sin x, x \in[-\pi, \pi]$, defines a Hilbert space isomorphism from $L^{2}(T, v \mathrm{~d} t) \equiv$ $L^{2}\left([-\pi, \pi], \sin ^{2} x \mathrm{~d} x\right)$ onto $L^{2}(T, \mathrm{~d} t) \equiv L^{2}[-\pi, \pi]$ which maps the subspace $\mathcal{H}$ of $W$-invariants onto the subspace of odd functions. The Hamiltonian is given formally by the same expression as $H_{2}$. A core for this operator is given by the odd $2 \pi$-periodic $C^{\infty}$-functions on $\mathbb{R}$. Hence, this operator describes a planar pendulum of mass $m=\frac{1}{2 \beta^{2}}$ and ratio of gravitational acceleration by length given by $\frac{v}{\hbar^{2} \beta^{2}}$ with the constraint that among the states of the pendulum only the odd ones emerge. Finally, restriction to $[0, \pi]$ defines a Hilbert space isomorphism from the subspace of $L^{2}[-\pi, \pi]$ of odd functions onto $L^{2}[0, \pi]$ that carries the Hamiltonian of the planar pendulum to $H_{2}$. Hence, we arrive again at the square potential with cosine potential inside. By construction, the resulting isomorphism $\mathcal{H} \equiv L^{2}(T, v \mathrm{~d} t)^{W} \rightarrow L^{2}[0, \pi]$ coincides with $\Gamma$.

Remark 5.2. The relation between our system and the quantum planar pendulum is the quantum counterpart of the observation made above that the reduced classical phase space of our system is isomorphic, as a stratified symplectic space, to that of a spherical pendulum, constrained to move with zero angular momentum, reduced relative to rotations about the vertical axis. This system is manifestly equivalent to that of a planar pendulum reduced relative to reflection about the vertical axis.

Now we discuss briefly the extension problem which arises in this context. Naive quantization after reduction on $\mathrm{T}^{*} K$ fails because of the presence of singularities on $\mathcal{P}$. The part of $\mathrm{T}^{*} K$ to which regular cotangent bundle reduction applies is the cotangent bundle of the unreduced principal stratum $K \backslash\{ \pm \mathbb{1}\}$. On this part, symplectic reduction leads to the cotangent bundle of the quotient manifold, i.e., of the principal stratum $\mathcal{X}_{1}$ but, beware, $\mathrm{T}^{*} \mathcal{X}_{1}$ is a proper subset of the principal stratum $\mathcal{P}_{1}$ of the reduced phase space $\mathcal{P}$ rather than being the entire stratum. In the parameterization of $\mathcal{X}$ chosen above, $\mathcal{X}_{1}$ corresponds to the open interval $] 0, \pi$. Since the parameterization is an isometry when scaled via $\beta$, canonical quantization of the kinetic energy then yields the symmetric operator

$$
\beta^{2} \frac{\mathrm{d}^{2}}{\mathrm{~d} x^{2}}
$$

on the Hilbert space $L^{2}[0, \pi]$ having as domain the compactly supported smooth functions on the open interval $] 0, \pi$ [. This leads to a naive quantization procedure away from the singularities of $\mathcal{X}$.

To arrive at a well-defined quantum theory of the entire system including the singular subset $\mathcal{X}_{0}$, one faces the problem of determining the self-adjoint extensions of the operator (5.3), each of which defines a different quantum theory, and to isolate one of these extensions as the 'correct' one. Thus, among the different extensions, one has to pick one according to the boundary conditions imposed on the wave functions and the physical interpretation of the theory will depend on the choice of boundary conditions. This is the 
problem studied in [17] in the situation where the classical configuration space is a cone over a Riemannian manifold; see also [14] and [37], where related questions are discussed under a more general perspective. When the classical configuration space arises by reduction, the extension problem does not really arise, though, since by reduction after quantization the kinetic energy operator is uniquely determined. This was already observed in [57] in the context of quantization by Rieffel induction. Indeed, in our situation, up to the shift by $\beta^{2}$ which, in the case of (5.3), can be obtained by the metaplectic correction, $\Delta_{2}$ is a self-adjoint extension of (5.3). According to Proposition 5.1, this is the Friedrichs extension.

To conclude we speculate that some deeper insight into quantization after reduction will, perhaps, make the kinetic energy operator unique in general as well.

5.2. Eigenvalues and eigenstates. For $v=0$, i. e., in the strong coupling limit, in view of (3.15) and (4.3), the energy eigenvalues are given by

$$
E_{n, \nu=0}=\frac{\hbar^{2}}{2} \varepsilon_{n}=\frac{\hbar^{2} \beta^{2}}{2} n(n+2),
$$

and the corresponding normalized eigenfunctions are given by the characters $\chi_{n}$. To solve the eigenvalue problem for nonvanishing $v$ we carry $H$ via $\Gamma$ to $H_{2}$. Let

$$
\tilde{v}=\frac{v}{\hbar^{2} \beta^{2}} \equiv \frac{1}{\hbar^{2} \beta^{2} g^{2}} .
$$

According to (5.1) and (5.2), on the core $\mathrm{D}_{2}$ of $\mathrm{H}_{2}$,

$$
H_{2}=-\frac{\hbar^{2} \beta^{2}}{2}\left(\frac{\mathrm{d}^{2}}{\mathrm{~d} x^{2}}+2 \tilde{v} \cos (x)+(1-3 \tilde{v})\right),
$$

and so the stationary Schrödinger equation for $\mathrm{H}_{2}$ reads

$$
\left(\frac{\mathrm{d}^{2}}{\mathrm{~d} x^{2}}+2 \tilde{v} \cos (x)+\left(\frac{2 E}{\hbar^{2} \beta^{2}}+1-3 \tilde{v}\right)\right) \psi(x)=0,
$$

$E$ being the desired eigenvalue and $\psi \in D_{2}$ the corresponding eigenfunction. The change of variable $y=(x-\pi) / 2$ leads to the Mathieu equation

$$
\frac{\mathrm{d}^{2}}{\mathrm{~d} y^{2}} f(y)+(a-2 q \cos (2 y)) f(y)=0,
$$

where

$$
a=\frac{8 E}{\hbar^{2} \beta^{2}}+4-12 \tilde{v}, \quad q=4 \tilde{v},
$$

and $f$ is a Whitney smooth function on the interval $[-\pi / 2,0]$ satisfying the boundary conditions

$$
f(-\pi / 2)=f(0)=0 .
$$

For the theory of the Mathieu equation and its solutions, called Mathieu functions, see $[7,46,47]$. All we need is this: for certain characteristic values of the parameter $a$, depending analytically on $q$ and usually being denoted by $b_{2 n+2}(q), n=0,1,2, \ldots$, solutions 
satisying (5.7) exist. Given $a=b_{2 n+2}(q)$, the corresponding solution is unique up to a complex factor and can be chosen to be real-valued. It is usually denoted by $\operatorname{se}_{2 n+2}(y ; q)$, where 'se' stands for sine elliptic. For given $\tilde{v} \geq 0$, let the vectors $\xi_{n} \in \mathcal{H}$ be defined by

$$
\left(\Gamma \xi_{n}\right)(x)=(-1)^{n+1} \sqrt{2}\left(\operatorname{se}_{2 n+2}\left(\frac{x-\pi}{2} ; 4 \tilde{v}\right)\right), \quad n=0,1,2, \ldots
$$

Since $\operatorname{se}_{2 n+2}(y ; 0)=\sin ((2 n+2) y)$ the factor $(-1)^{n+1}$ ensures that, for $\tilde{v}=0$, we get $\xi_{n}=\chi_{n}$ exactly and not only up to a sign.

Theorem 5.3. For any $\tilde{v} \geq 0$, the vectors $\xi_{n} \in \mathcal{H}, n=0,1,2, \ldots$, form an orthonormal basis of eigenvectors of $H$. The corresponding eigenvalues are non-degenerate. They are given by

$$
E_{n}=\frac{\hbar^{2} \beta^{2}}{2}\left(\frac{b_{2 n+2}(4 \tilde{v})}{4}+3 \tilde{v}-1\right)
$$

Proof. This follows at once from the fact that, for any value of the parameter $q$, the functions $\sqrt{2} \operatorname{se}_{2 n+2}(y ; q), n=0,1,2, \ldots$, form an orthonormal basis in $L^{2}[-\pi / 2,0]$, see $[2, \S 20.5]$. Moreover, the characteristic values satisfy $b_{2}(q)<b_{4}(q)<b_{6}(q)<\cdots$, see $[2, \S 20.2]$. Hence, for any value of $\tilde{v}$ we have $E_{0}<E_{1}<E_{2}<\cdots$.

Fig. 5 shows the energy eigenvalues $E_{n}$ and the level separation $E_{n+1}-E_{n}$ for $n=$ $0, \ldots, 8$ as functions of $\tilde{v}$. The transition energy values manifestly reverse their order as $v$ increases. Fig. 6 displays the images of eigenfunctions $\xi_{n}, n=0, \ldots, 3$, under $\Gamma$ (i.e., the rescaled and shifted Mathieu functions themselves), for $\tilde{v}=0,3,6,12,24$. The plots have been generated by means of the built-in Mathematica functions Mathieus and MathieucharacteristicB.
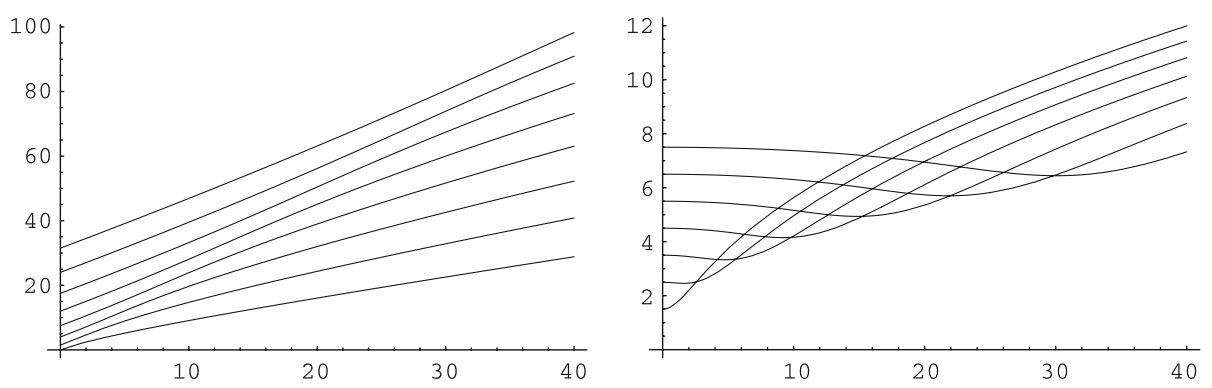

Fig. 5. Energy eigenvalues (left) $E_{n}$ and transition energy values $E_{n+1}-E_{n}$ (right) for $n=0, \ldots, 7$ in units of $\hbar^{2} \beta^{2}$ as functions of $\tilde{v}$

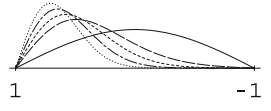

$\xi_{0}$

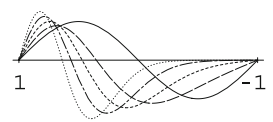

$\xi_{1}$

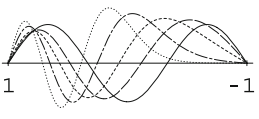

$\xi_{2}$

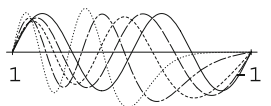

$\xi_{3}$

Fig. 6. Images of the energy eigenfunctions $\xi_{0}, \ldots, \xi_{3}$, under $\Gamma$, for $\tilde{v}=0$ (continuous line), 3 (long dash), 6 (short dash), 12 (alternating short-long dash), 24 (dotted line) 
Remark 5.4. The Schrödinger equation for the planar pendulum is solved in an analogous way [12]. From the discussion in Subsect. 5.1 it follows that the only difference is that in the case of the pendulum, the function $f$ in (5.5) can be any $\pi$-periodic smooth function on $\mathbb{R}$. Then, in addition to the family of $\pi$-periodic odd solutions given by the functions $\operatorname{se}_{2 n+2}(y ; q)$ and their characteristic values $b_{2 n+2}(q)$ there is a family of $\pi$-periodic even solutions which are usually denoted by $c_{2 n+2}$ (for 'cosine elliptic'). The corresponding characteristic values are usually denoted by $a_{2 n+2}(q)$. For any value of $q, a_{2}(q)<b_{2}(q)<a_{4}(q)<b_{4}(q)<\cdots$. Thus, precisely every second eigenstate of the planar pendulum emerges in our system. In particular, the ground state of our system does not correspond to the ground state but to the first excited state of the planar pendulum.

Remark 5.5. According to Remark 2.1, Theorem 5.3 yields the solutions to the stationary Schrödinger equation for quantum Yang-Mills theory on $S^{1}$ when the self-interaction is described by the potential in (2.5). In particular, in this simple model we have constructed the vacuum and all excited states, for arbitrary values of the coupling constant.

\section{Expectation Values of the Costratification Orthoprojectors for SU(2)}

The most elementary observables associated with the costratification are the orthoprojectors $\Pi_{i}$ onto, respectively, the subspaces $\mathcal{H}_{i}, i= \pm, 0$. The expectation value of $\Pi_{i}$ in a state $\psi$ yields the probability that the system prepared in this state is measured in the subspace $\mathcal{H}_{i}$. We determine the expectation values of $\Pi_{i}$ in the energy eigenstates, i. e.,

$$
P_{i, n}:=\left\langle\xi_{n} \mid \Pi_{i} \xi_{n}\right\rangle=\left\|\Pi_{i} \xi_{n}\right\|^{2}, \quad i=0, \pm .
$$

Then, we derive approximations for these expectation values for strong and weak coupling.

6.1. Expectation values. According to (4.14),

$$
P_{ \pm, n}=\left|\left\langle\xi_{n} \mid \psi_{ \pm}\right\rangle\right|^{2}, \quad P_{0, n}=\left|\left\langle\xi_{n} \mid \psi_{\mathrm{g}}\right\rangle\right|^{2}+\left|\left\langle\xi_{n} \mid \psi_{\mathrm{u}}\right\rangle\right|^{2}
$$

As $\operatorname{se}_{2 n+2}$ is odd and $\pi$-periodic, it can be expanded as

$$
\operatorname{se}_{2 n+2}(y ; q)=\sum_{k=0}^{\infty} B_{2 k+2}^{2 n+2}(q) \sin ((2 k+2) y),
$$

where $B_{2 k+2}^{2 n+2}(q)$ refers to the Fourier coefficients. The Fourier coefficients satisfy certain recurrence relations, see $[2, \S 20.2]$. Using (4.24), we find

$$
\left\langle\xi_{n} \mid k\right\rangle=(-1)^{n+k} B_{2 k+2}^{2 n+2}(4 \tilde{v}) .
$$

Then (4.11)-(4.13) yield

$$
\begin{aligned}
\left\langle\xi_{n} \mid \psi_{+}\right\rangle & =\frac{(-1)^{n}}{N} \sum_{k=0}^{\infty}(-1)^{k}(k+1) \mathrm{e}^{-\hbar \beta^{2}(k+1)^{2} / 2} B_{2 k+2}^{2 n+2}(4 \tilde{v}), \\
\left\langle\xi_{n} \mid \psi_{-}\right\rangle & =\frac{(-1)^{n}}{N} \sum_{k=0}^{\infty}(k+1) \mathrm{e}^{-\hbar \beta^{2}(k+1)^{2} / 2} B_{2 k+2}^{2 n+2}(4 \tilde{v}), \\
\left\langle\xi_{n} \mid \psi_{\mathrm{g}}\right\rangle & =\frac{(-1)^{n}}{N_{\mathrm{g}}} \sum_{k=0}^{\infty}(2 k+1) \mathrm{e}^{-\hbar \beta^{2}(2 k+1)^{2} / 2} B_{4 k+2}^{2 n+2}(4 \tilde{v}), \\
\left\langle\xi_{n} \mid \psi_{\mathrm{u}}\right\rangle & =-\frac{(-1)^{n}}{N_{\mathrm{u}}} \sum_{k=0}^{\infty}(2 k+2) \mathrm{e}^{-\hbar \beta^{2}(2 k+2)^{2} / 2} B_{4 k+4}^{2 n+2}(4 \tilde{v}) .
\end{aligned}
$$



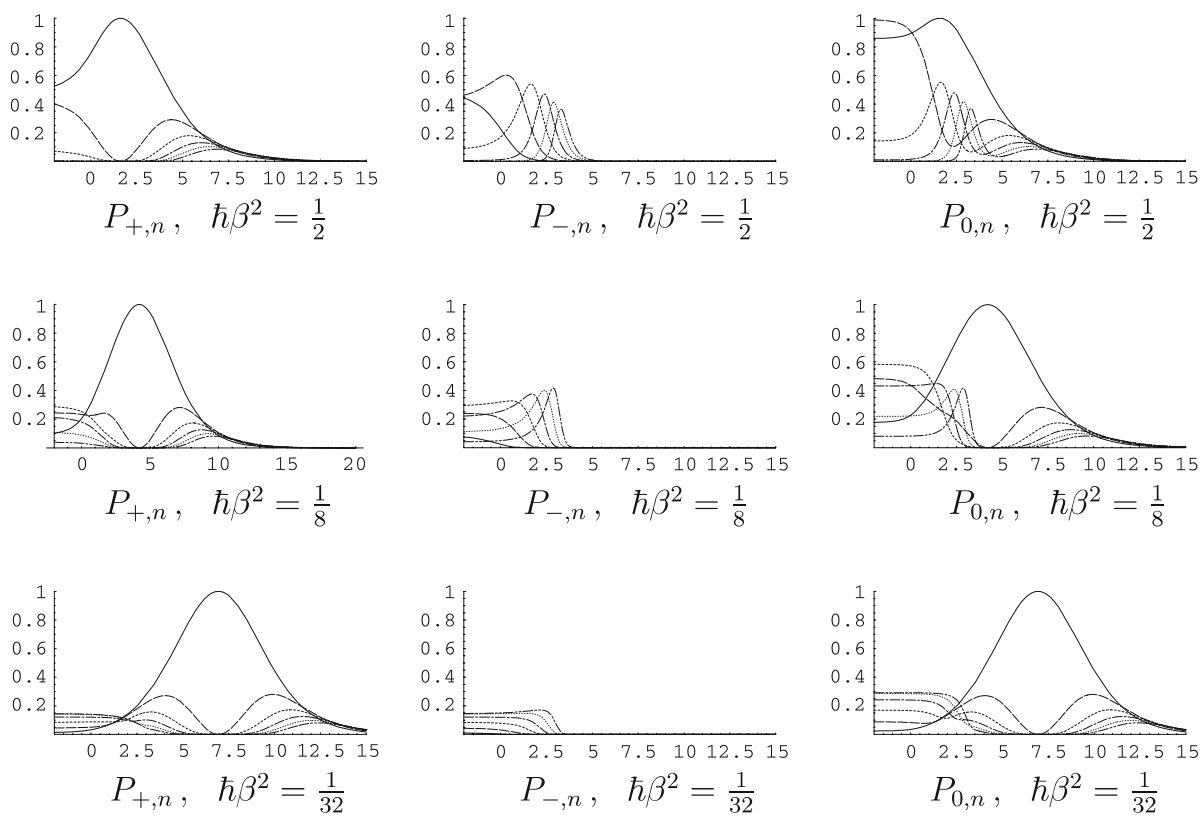

Fig. 7. Expectation values $P_{+, n}, P_{-, n}$ and $P_{0, n}$ for $n=0$ (continuous line), 1 (long dash), 2 (short dash), 3 (long-short dash), 4 (dotted line) and 5 (long-short-short dash), plotted over $\log \tilde{v}$ for $\hbar \beta^{2}=\frac{1}{2}, \frac{1}{8}, \frac{1}{32}$

Together with (6.1), this yields formulas for the $P_{i, n}$ 's, $i=0, \pm$. We do not spell them out, since they do not lead to significant simplification. The functions $P_{i, n}$ depend on the parameters $\hbar, \beta^{2}$ and $v$ only via the combinations $\hbar \beta^{2}$ and $\tilde{v}=v /\left(\hbar^{2} \beta^{2}\right)$. Fig. 7 displays the $P_{i, n}, n=0, \ldots, 5$, as functions of $\tilde{v}$ for three specific values of $\hbar \beta^{2}$, thus treating $\tilde{v}$ and $\hbar \beta^{2}$ as independent parameters. This is appropriate for the discussion of the dependence of $P_{i, n}$ on the coupling parameter $g$ for fixed values of $\hbar$ and $\beta^{2}$. The plots have been generated by Mathematica through numerical integration.

Perhaps the most impressive feature is the dominant peak of $P_{+, 0}$ which is enclosed by less prominent maxima of the other $P_{+, n}$ and moves to higher $\tilde{v}$ when $\hbar \beta^{2}$ decreases. In other words, for a certain value of the coupling constant, the state $\psi_{+}$which spans $\mathcal{H}_{+}$seems to coincide almost perfectly with the ground state. If the two states coincided completely then (6.2) would imply that, for a certain value of $q$, the coefficients $B_{2 k+2}^{2 n+2}(q)$ would be given by $(-1)^{n+k} \frac{1}{N}(k+1) \mathrm{e}^{-\hbar \beta^{2}(k+1)^{2} / 2}$. However, this is not true; the latter expressions do not satisfy the recurrence relations valid for the coefficients $B_{2 k+2}^{2 n+2}(q)$ for any value of $q$. Another interesting phenomenon is that, for decreasing $\hbar \beta^{2}$, the maxima of $P_{-, n}$ move to lower $\tilde{v}$ and the subsequent descent becomes steeper.

Next, we will derive approximations for the $P_{i, n}$ 's for small and large values of $\tilde{v}$. When $\hbar$ and $\beta$ are fixed, this corresponds to strong and weak coupling, as appropriate. The strong coupling approximation will provide a resolution of the first crossings of the graphs of the $P_{i, n}$. The weak coupling approximation will allow us to analyze the position and the height of the dominant peak of $P_{+, 0}$ as well as of the subsequent maxima of the other $P_{+, n}$ 's. A detailed study of the maxima of the $P_{-, n}$ 's and of the behaviour of the $P_{+, n}$ 's in the intermediate region between strong and weak coupling remains as a future task. 
6.2. Strong coupling approximation. In the region of strong coupling, i. e., for large $g, \tilde{v}$ is small, at least when the parameter $\hbar \beta^{2}$ is fixed. Power series expansions for the characteristic values $b_{2 n+2}(q)$ in $q$ about $q=4 \tilde{v}=0$ can be found, e. g., in [2, §20.2.25]. They immediately provide expansions for the energy eigenvalues. We do not spell out the latter here, because we are merely interested in approximations of the expectation values $P_{i, n}, i= \pm, 0$. Quadratic approximations for the Fourier coefficients $B_{2 k+2}^{2 n+2}(q)$ in $q$ can be read off from $[47, \S 2.25,(42)]$ : For the central coefficients, this yields

$$
\begin{aligned}
B_{2}^{2}(4 \tilde{v}) & =1-\frac{1}{18} \tilde{v}^{2}+O\left(\tilde{v}^{3}\right) \\
B_{2 n+2}^{2 n+2}(4 \tilde{v}) & =1-\frac{(2 n+2)^{2}+1}{2\left((2 n+2)^{2}-1\right)^{2}} \tilde{v}^{2}+O\left(\tilde{v}^{3}\right), \quad n \geq 1 .
\end{aligned}
$$

For the next-to-central coefficients,

$$
\begin{aligned}
& B_{2 n}^{2 n+2}(4 \tilde{v})=\frac{1}{(2 n+1)} \tilde{v}+O\left(\tilde{v}^{3}\right), B_{2 n+4}^{2 n+2}(4 \tilde{v})=-\frac{1}{(2 n+3)} \tilde{v}+O\left(\tilde{v}^{3}\right), \\
& B_{2 n-2}^{2 n+2}(4 \tilde{v})=\frac{1}{4 n(2 n+1)} \tilde{v}^{2}+O\left(\tilde{v}^{3}\right), B_{2 n+6}^{2 n+2}(4 \tilde{v})=\frac{1}{2(2 n+3)(2 n+4)} \tilde{v}^{2}+O\left(\tilde{v}^{3}\right), \quad n \geq 0 .
\end{aligned}
$$

All the other coefficients are of order $O\left(\tilde{v}^{3}\right)$. Using (6.1) and (6.3)-(6.6) we obtain

$$
P_{ \pm, n}=\frac{A_{n, 0}^{2}}{N^{2}} \pm \frac{2 A_{n, 0} A_{n, 1}}{N^{2}} \tilde{v}+\frac{A_{n, 1}^{2}+A_{n, 0} A_{n, 2}}{N^{2}} \tilde{v}^{2}+O\left(\tilde{v}^{3}\right)
$$

and, for $n$ even, we get

$$
P_{0, n}=\frac{A_{n, 0}^{2}}{N_{\mathrm{g}}^{2}}+\left(\frac{A_{n, 1}^{2}}{N_{\mathrm{u}}^{2}}+\frac{A_{n, 0} A_{n, 2}}{N_{\mathrm{g}}^{2}}\right) \tilde{v}^{2}+O\left(\tilde{v}^{3}\right),
$$

whereas, for $n$ odd, in this expression, one has to interchange $N_{\mathrm{g}}$ and $N_{\mathrm{u}}$. The coefficients are

$$
\begin{aligned}
A_{n, 0}= & (n+1) \mathrm{e}^{-\hbar \beta^{2}(n+1)^{2} / 2}, \quad n \geq 0, \\
A_{n, 1}= & \frac{(n+2) \mathrm{e}^{-\hbar \beta^{2}(n+2)^{2} / 2}}{2 n+3}-\frac{n \mathrm{e}^{-\hbar \beta^{2} n^{2} / 2}}{2 n+1}, \quad n \geq 0, \\
A_{0,2}= & \frac{\mathrm{e}^{-9 \hbar \beta^{2} / 2}}{4}-\frac{\mathrm{e}^{-\hbar \beta^{2} / 2}}{9}, \\
A_{n, 2}= & \frac{(n-1) \mathrm{e}^{-\hbar \beta^{2}(n-1)^{2} / 2}}{2 n(2 n+1)}+\frac{(n+3) \mathrm{e}^{-\hbar \beta^{2}(n+3)^{2} / 2}}{(2 n+3)(2 n+4)} \\
& -(n+1) \mathrm{e}^{-\hbar \beta^{2}(n+1)^{2} / 2}\left(\frac{1}{(2 n+1)^{2}}+\frac{1}{(2 n+3)^{2}}\right), \quad n \geq 1 .
\end{aligned}
$$

For $\hbar \beta^{2}=\frac{1}{8}$, plots of the quadratic approximations of the $P_{i, n}$ 's, $i= \pm, 0$, are shown in Fig. 8, for $n=0, \ldots, 5$ and $\tilde{v}$ ranging between 0 and 0.2 . Here the approximation has a relative error of less than 0.01 . The plots yield, in particular, a resolution of the first crossings of the graphs of the $P_{i, n}$ 's in the bottom line of Fig. 7.

For very strong coupling, the state $\xi_{2}$ rather than the ground state has the highest probability to be measured in $\mathcal{H}_{+}$. In fact the ground state is excelled by all $\xi_{n}$ with 

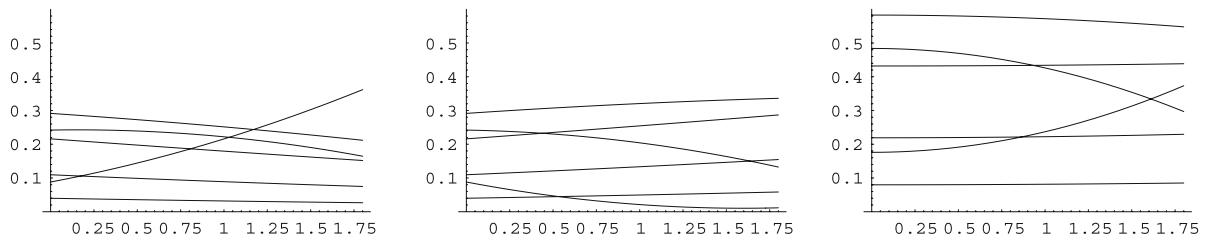

Fig. 8. Quadratic approximations for $P_{+, n}, P_{-, n}, P_{0, n}$ (from left to right), $n=0, \ldots, 5, \hbar \beta^{2}=\frac{1}{8}$, plotted over $\tilde{v}$

$n \leq 4$. (This follows of course directly from consideration of the case $v=0$, where $\xi_{n}=\chi_{n}$.) The precise order of the expectation values in this region is

$$
P_{i, 2} \geq P_{i, 1} \geq P_{i, 3} \geq P_{i, 4} \geq P_{i, 0} \geq P_{i, 5} \geq P_{i, 6} \geq \cdots, \quad i=0, \pm .
$$

On the other hand, the probabilities $P_{i, 0}$ of the ground state change most rapidly as $v$ increases. In particular, $P_{+, 0}$ has overtaken all other probabilities already for $v=0.2$.

6.3. Weak coupling approximation. Similarly to the approximation of a classical planar pendulum by a classical harmonic oscillator, for excitations that are small compared with the length of the pendulum, the quantum planar pendulum can be approximated by a quantum harmonic oscillator for energy values that are small compared with the range of the potential $[3,9,12,49]$. We use this procedure to obtain approximations for the energy eigenfunctions $\xi_{n}$ and, from these, approximations for the expectation values $P_{i, n}, i= \pm, 0$, for large $\tilde{v}$ and small $n$.

Consider the Schrödinger equation for $\mathrm{H}_{2}$ in (5.4). Making the change of variable $z=\sqrt[4]{\tilde{v}} x$ we obtain the equation

$$
\left(\frac{\mathrm{d}^{2}}{\mathrm{~d} z^{2}}+2 \tilde{v} \cos (z / \sqrt[4]{\tilde{\nu}})+\left(\frac{1}{\sqrt{\tilde{v}}}\left(\frac{2 E}{\hbar^{2} \beta^{2}}+1\right)-3 \sqrt{\tilde{v}}\right)\right) f(z)=0,
$$

where $f$ may be a Whitney smooth function on the interval $[0, \sqrt[4]{\tilde{v}} \pi]$ satisfying the boundary conditions $f(0)=f(\sqrt[4]{\tilde{v}} \pi)=0$. Replacing the cosine with its second order Taylor expansion we obtain the Schrödinger equation

$$
\left(\frac{\mathrm{d}^{2}}{\mathrm{~d} z^{2}}-z^{2}+2 \epsilon\right) f(z)=0
$$

of the harmonic oscillator with unit frequency, where

$$
\epsilon=\frac{1}{\sqrt{\tilde{v}}}\left(\frac{E}{\hbar^{2} \beta^{2}}+\frac{1}{2}\right)-\frac{\sqrt{\tilde{v}}}{2} .
$$

For large $\tilde{v}$ and small energies, the solutions of either equation are concentrated about $x=z / \sqrt[4]{\tilde{v}} \sim 0$. Under these circumstances, restriction to the interval $[0, \sqrt[4]{\tilde{\nu}} \pi]$ of solutions of (6.7) satisfying $f(0)=0$ yields satisfactory approximations for solutions of (5.4). The appropriate solutions of (6.7) are well known to be

$$
f(z)=H_{2 n+1}(z) \mathrm{e}^{-z^{2} / 2}, \quad \epsilon=2 n+\frac{3}{2}, \quad n=0,1,2, \ldots,
$$


where

$$
H_{2 n+1}(z)=\sum_{r=0}^{n} \frac{(-1)^{n+r}(2 n+1) !(2 z)^{2 r+1}}{(n-r) !(2 r+1) !}
$$

are the odd degree Hermite polynomials. Define vectors $\xi_{n}^{(\infty)} \in \mathcal{H}$ by

$$
\begin{aligned}
\left(\Gamma \xi_{n}^{(\infty)}\right)(x) & =(-1)^{n} N_{n}^{(\infty)} H_{2 n+1}(\sqrt[4]{\tilde{v}} x) \mathrm{e}^{-\sqrt{\tilde{v}} x^{2} / 2}, \\
N_{n}^{(\infty)} & =\frac{\pi^{1 / 4} \tilde{v}^{1 / 8}}{2^{n+1} \sqrt{(2 n+1) !}}
\end{aligned}
$$

where the choice of sign is dictated by that for the $\xi_{n}$ 's, see (5.8). The $\xi_{n}^{(\infty)}$ 's form a basis of $\mathcal{H}$. Substituting for $\epsilon$ the right-hand side of (6.8), we obtain the energy values

$$
E_{n}^{(\infty)}=\frac{\hbar^{2} \beta^{2}}{2}(\tilde{v}+(4 n+3) \sqrt{\tilde{v}}-1) .
$$

The $E_{n}^{(\infty)}$, s and the $\xi_{n}^{(\infty)}$, s yield approximations for the true energy eigenvalues $E_{n}$ and for the eigenfunctions $\xi_{n}$ of our model for large $\tilde{v}$ and small $n$. Note that the $\xi_{n}$ 's are neither orthogonal nor normalized, because the functions $\Gamma \xi_{n}^{(\infty)}$ are orthogonal over the interval $[0, \infty]$ rather than the interval $[0, \pi]$ and the normalization factor $N_{n}^{(\infty)}$ is therefore also chosen so that the functions are normalized over the interval $[0, \infty]$. The deviation from being orthonormal is however negligible for small $n$ and large $\tilde{\nu}$.

To compute the scalar products $\left\langle\chi_{k}, \xi_{n}^{(\infty)}\right\rangle$, we use (4.24) and (6.9) and move the upper bound of the resulting integral from $\pi$ to infinity, which is again justified for large $\tilde{v}$ and small $n$. The result is

$$
\left\langle\chi_{k}, \xi_{n}^{(\infty)}\right\rangle=\frac{2^{-n} \pi^{-1 / 4}}{\sqrt{(2 n+1) !}} \tilde{v}^{-1 / 8} H_{2 n+1}\left((k+1) \tilde{v}^{-1 / 4}\right) \mathrm{e}^{-(k+1)^{2} \tilde{v}^{-1 / 2} / 2} .
$$

This formula is also a consequence of (6.2) and the asymptotic expansion of the Fourier coefficients $B_{2 k+2}^{2 n+2}(q)$ for large $q$ given in [47, §2.333]. Using (6.11) and writing out the formula of the Hermite polynomials, we obtain

$$
\left\langle\psi_{i}, \xi_{n}^{(\infty)}\right\rangle=(-1)^{n} \frac{\sqrt{(2 n+1) !}}{2^{n} \pi^{1 / 4} N_{i}} \sum_{r=0}^{n} \frac{(-1)^{r} 2^{2 r+1} \tilde{v}^{-(4 r+3) / 8}}{(n-r) !(2 r+1) !} \Sigma_{i}^{r}\left(\frac{\hbar \beta^{2}+\tilde{v}^{-1 / 2}}{2}\right)
$$

where $i= \pm, \mathrm{g}, \mathrm{u}, N_{ \pm} \equiv N$, and

$$
\begin{array}{ll}
\Sigma_{+}^{r}(b)=\sum_{k=1}^{\infty} k^{2 r+2} \mathrm{e}^{-b k^{2}}, & \Sigma_{-}^{r}(b)=\sum_{k=1}^{\infty}(-1)^{k+1} k^{2 r+2} \mathrm{e}^{-b k^{2}} . \\
\Sigma_{\mathrm{g}}^{r}(b)=\sum_{k \text { odd }} k^{2 r+2} \mathrm{e}^{-b k^{2}}, & \Sigma_{\mathrm{u}}^{r}(b)=\sum_{k \text { even }} k^{2 r+2} \mathrm{e}^{-b k^{2}} .
\end{array}
$$


Expressing the sums in terms of the theta constant $\theta_{3}$, see (4.15), we obtain

$$
\begin{aligned}
& \Sigma_{+}^{r}(b)=\frac{1}{2} \frac{\mathrm{d}^{r+1}}{\mathrm{~d}(-b)^{r+1}} \theta_{3}\left(\mathrm{e}^{-b}\right), \quad \Sigma_{-}^{r}(b)=\Sigma_{+}^{r}(b)-2^{2 r+3} \Sigma_{+}^{r}(4 b), \\
& \Sigma_{\mathrm{g}}^{r}(b)=\Sigma_{+}^{r}(b)-4^{r+1} \Sigma_{+}^{r}(4 b), \quad \Sigma_{\mathrm{u}}^{r}(b)=4^{r+1} \Sigma_{+}^{r}(4 b) .
\end{aligned}
$$

Substituting in (6.12), for $\Sigma_{i}^{r}$, the right-hand side of each of these identities as appropriate and taking the square, we arrive at the harmonic oscillator approximations of $P_{ \pm, n}$ and $P_{0, n}$. These approximations are hard to handle, however, as they contain higher derivatives of the theta constant w.r.t. the nome. Instead, we use the approximation

$$
\theta_{3}\left(e^{-y}\right)=\sqrt{\pi} y^{-1 / 2}+\cdots,
$$

valid for small $y$ and hence for small $\hbar \beta^{2}$ and large $\tilde{v}$. Even for $y=1$, this approximation has a relative error of only $10^{-4}$. In this approximation,

$$
\Sigma_{+}^{r}(b)=\sqrt{\pi} \frac{(2 r+1) !}{4^{r+1} r !} b^{-(2 r+3) / 2}, \quad \Sigma_{-}^{r}(b)=0, \quad \Sigma_{\mathrm{g}}^{r}(b)=\Sigma_{\mathrm{u}}^{r}(b)=\frac{1}{2} \Sigma_{+}^{r}(b),
$$

and

$$
N=\sqrt{\Sigma_{+}^{2}\left(\hbar \beta^{2}\right)}=\frac{\pi^{1 / 4}}{\sqrt{2}}\left(\hbar \beta^{2}\right)^{-3 / 4}, \quad N_{\mathrm{g}}=N_{\mathrm{u}}=\frac{1}{\sqrt{2}} N .
$$

In particular, $\mathcal{H}_{+}$and $\mathcal{H}_{-}$appear to be orthogonal. Moreover, (6.12) yields $P_{-, n}=0$ and $P_{0, n}=P_{+, n}$, so that it suffices to determine $P_{+, n}$. Inserting $\Sigma_{+}^{r}$ from (6.14) into (6.12) and writing

$$
\tau=\sqrt{\hbar} \beta \tilde{v}^{1 / 4} \equiv \sqrt{\frac{\beta}{g}}
$$

we obtain the identity

$$
\left\langle\psi_{+}, \xi_{n}^{(\infty)}\right\rangle=(-1)^{n} \frac{\sqrt{(2 n+1) !}}{2^{n}} \sum_{r=0}^{n} \frac{(-1)^{r} 2^{(2 r+3) / 2}}{r !(n-r) !} \frac{\tau^{3 / 2}}{\left(\tau^{2}+1\right)^{(2 n+3) / 2}} .
$$

Taking the sum yields

$$
\left\langle\psi_{+}, \xi_{n}^{(\infty)}\right\rangle=(-1)^{n} \frac{\sqrt{(2 n+1) !}}{2^{n} n !}\left(\frac{2 \tau}{\tau^{2}+1}\right)^{3 / 2}\left(\frac{\tau^{2}-1}{\tau^{2}+1}\right)^{n} .
$$

Hence, in the harmonic oscillator approximation and the approximation of $\theta_{3}$ by (6.13), the expectation values $P_{+, n}$ are given by the rational functions

$$
P_{+, n}^{(\infty)}(\tau)=\frac{(2 n+1) !}{4^{n}(n !)^{2}}\left(\frac{2 \tau}{\tau^{2}+1}\right)^{3}\left(\frac{\tau^{2}-1}{\tau^{2}+1}\right)^{2 n}
$$

It is interesting to note that, in this approximation, $P_{+, n}$ depends on the parameters $\hbar$, $\beta$ and $v$ only through the ratio $\beta / g$. Fig. 9 shows plots of $P_{+, n}^{(\infty)}$ and $P_{+, n}$ as functions 

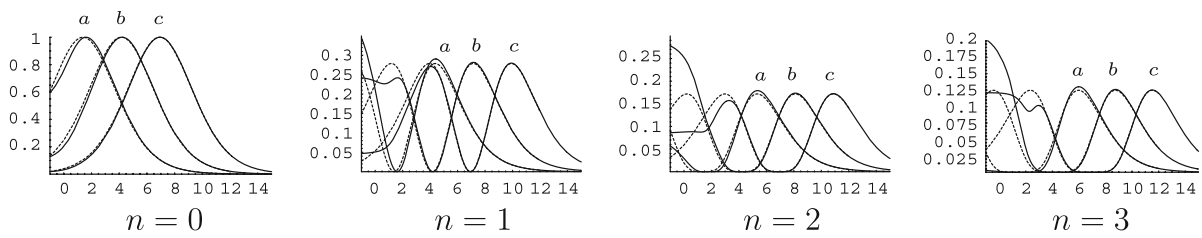

Fig. 9. Exact values of $P_{+, n}$ (continuous lines) and approximations $P_{+, n}^{(\infty)}$ (dashed lines) as functions of $\tilde{v}$ on a logarithmic scale for $\hbar \beta^{2}=\frac{1}{2}(a), \frac{1}{8}(b), \frac{1}{32}(c)$ and $n=0, \ldots, 3$

of $\tilde{v}$ on a logarithmic scale for $\hbar \beta^{2}=1, \frac{1}{2}, \frac{1}{8}$ and $n=0, \ldots, 3$. We see that for sufficiently small values of $\hbar \beta^{2}$ and sufficiently small $n$ the approximation of $P_{+, n}$ by $P_{+, n}^{(\infty)}$ is already satisfactory in the region of the dominant maximum of $P_{+, 0}$ and even more so for larger $\tau$. Hence, this approximation can be used to study the behaviour of $P_{+, n}$ in this region.

Moreover, we claim that this approximation is consistent in the sense that, for any $\tau>0$,

$$
P_{+, n}^{(\infty)}(\tau) \geq 0, \quad \sum_{n=0}^{\infty} P_{+, n}^{(\infty)}(\tau)=1
$$

Indeed,

$$
\sum_{n=0}^{\infty} P_{+, n}^{(\infty)}(\tau)=\left(\frac{2 \tau}{\tau^{2}+1}\right)^{3} \sum_{n=0}^{\infty} \frac{(2 n+1) !}{4^{n}(n !)^{2}}\left(\frac{\tau^{2}-1}{\tau^{2}+1}\right)^{2 n} .
$$

Recall that the function $y \mapsto(1-y)^{-3 / 2}$ has the Taylor series

$$
(1-y)^{-3 / 2}=\sum_{n=0}^{\infty} \frac{(2 n+1) !}{4^{n}(n !)^{2}} y^{n},
$$

and this series is absolutely convergent for $|y|<1$. Replacing $y$ with $\left(\tau^{2}-1\right)^{2} /\left(\tau^{2}+1\right)^{2}$, where $\tau>0$, we deduce that the approximation is consistent in the asserted sense.

We determine the extremal points of $P_{+, n}^{(\infty)}$ on the positive semiaxis. For $n=0$,

$$
\frac{\mathrm{d}}{\mathrm{d} \tau} P_{+, 0}^{(\infty)}(\tau)=\frac{24 \tau^{2}\left(1-\tau^{2}\right)}{\left(\tau^{2}+1\right)^{4}}
$$

Hence, at $\tau=1, P_{+, 0}^{(\infty)}$ has a maximum, the maximal value being

$$
P_{+, 0}^{(\infty)}(\tau=1)=1
$$

This means that, for coupling constant $g=\beta$, up to the approximations we have made, the state $\psi_{+}$spanning $\mathcal{H}_{+}$coincides with the ground state. In particular, the state $\psi_{+}$ is then approximately stationary. As remarked in Subsect. 6.1, the coincidence holds only in the approximation and is not exact though. A physical interpretation of this phenomenon has still to be found. For $n \geq 1$,

$$
\frac{\mathrm{d}}{\mathrm{d} \tau} P_{+, n}^{(\infty)}(\tau)=-\frac{(2 n+1) !}{2^{2 n-3}(n !)^{2}} \frac{\tau^{2}\left(\tau^{2}-1\right)^{2 n-1}}{\left(\tau^{2}+1\right)^{2 n+4}}\left(3 \tau^{4}-(8 n+6) \tau^{2}+3\right) .
$$


Hence, $P_{+, n}^{(\infty)}$ has maxima at

$$
\tau_{ \pm}=\sqrt{\frac{4 n+3 \pm 2 \sqrt{4 n^{2}+6 n}}{3}}
$$

and a minimum at $\tau=1$. The first maximum, $\tau_{-}$, lies in a region where the approximation is reliable only for very small values of $\hbar \beta^{2}$, see Fig. 9. For increasing $n, \tau_{-}$ approaches $\tau=1$ from below and $\tau_{+}$moves towards larger values of $\tau$. The maximal values of $P_{+, n}^{(\infty)}$ are

$$
P_{+, n}^{(\infty)}\left(\tau_{ \pm}\right)=\frac{3^{3 / 2}(2 n+1) !}{2^{2 n-3}(n !)^{2}} \frac{\left(4 n+3 \pm 2 \sqrt{4 n^{2}+6 n}\right)^{3 / 2}\left(4 n \pm 2 \sqrt{4 n^{2}+6 n}\right)^{2 n}}{\left(4 n+6 \pm 2 \sqrt{4 n^{2}+6 n}\right)^{2 n+3}} .
$$

These values are independent of the parameters $\hbar, \beta$ and $v$ and decrease for increasing $n$.

In the minimum $\tau=1, P_{+, n}^{(\infty)}$ vanishes. This is consistent with what we have found for $P_{+, 0}^{(\infty)}$. The order of contact of $P_{+, n}^{(\infty)}$ with the real axis is $2 n$. This order of contact is reflected in a broadening of the valley between the two maxima, see Fig. 9.

\section{Outlook}

There is still more to say about the case of SU(2). The expectation values $P_{ \pm, n}$ and $P_{0, n}$ in the region between the strong and weak coupling approximations and the dynamics relative to the costratified structure remain to be studied. The exploration of that dynamics will rely on a detailed investigation of the probability flow into and out of the subspaces $\mathcal{H}_{ \pm}, \mathcal{H}_{0}$.

The next step is the construction of the costratified Hilbert space and the subsequent analysis of various physical quantities for SU(3). Here, the orbit type stratification of the reduced phase space has a 4-dimensional stratum, a 2-dimensional stratum, and three isolated points. Thereafter the construction remains to be extended to an arbitrary lattice.

Finally, the costratified Hilbert space structure exploited in this paper implements the stratification of the reduced classical phase space on the level of states but leaves open the question what the stratification might signify for the quantum observables, a question to be clarified in the future. Then the physical role of this stratification can, perhaps, be studied in more realistic models like lattice QCD [36,38,39].

Acknowledgement. The authors would like to express their gratitude to Szymon Charzyński, Heinz-Dietrich Doebner, Alexander Hertsch, Jerzy Kijowski and Konrad Schmüdgen for the stimulus of conversation, to Brian Hall for pointing out the relationship between the states spanning $\mathcal{H}_{ \pm}$and coherent states, to Christian Fleischhack for hints at Thiemann's work on the overlap of coherent states, and to Jim Stasheff for a number of comments which helped improve the exposition. J. H. and M. S. acknowledge funding by the German Research Council (DFG) under contract Le 758/22-1 and contract RU692/3, respectively.

\section{References}

1. Abraham, R., Marsden, J.E.: Foundations of mechanics. Reading, MA: Benjamin/Cummings Publishing Co., Inc., Reading, Mass., 1978

2. Abramowitz, M., Stegun, I.A.: Handbook of Mathematical Functions (abridged edition). Frankfurt am Main: Verlag Harri Deutsch, 1984 
3. Aldrovandi, R., Leal Ferreira, P.: Quantum pendulum. Amer. J. Phys 48, 660-664 (1980)

4. Arms, J.M., Cushman, R., Gotay, M.J.: A universal reduction procedure for Hamiltonian group actions. The geometry of Hamiltonian systems. In: Ratiu, T. (ed.), MSRI Publ 20, Berlin-Heidelberg, New York: Springer 1991, pp. 33-51

5. Arms, J.M., Marsden, J.E., Moncrief, V.: Symmetry and bifurcation of moment mappings. Commun. Math. Phys. 78, 455-478 (1981)

6. Arms, J.M., Marsden, J.E., Moncrief, V.: The structure of the space of solutions of Einstein's equations. II. Several Killing fields and the Einstein-Yang-Mills equations. Ann. Phys. 144(1), 81-106 (1982)

7. Arscott, F.M.: Periodic Differential Equations. An Introduction to Mathieu, Lamé, and Allied Functions. London:Pergamon Press, 1964

8. Asorey, M., Falceto, F., López, J.L., Luzón, G.: Nodes, monopoles and confinement in (2+1)-dimensional gauge theories. Phys. Lett. B 349, 125-130 (1995)

9. Baker, G.L., Blackburn, J.A., Smith, H.J.T.: The quantum pendulum: Small and large. Amer. J. Phys. 70, 525-531 (2002)

10. Charzyński, S., Kijowski, J., Rudolph, G., Schmidt, M.: On the stratified classical configuration space of lattice QCD. J. Geom. Phys. 55, 137-178 (2005)

11. Charzyński, S., Rudolph, G., Schmidt, M.: On the topological structure of the stratified classical configuration space of lattice QCD. J. Geom. Phys. 58, 1607-1623 (2008)

12. Condon, E.U.: The physical pendulum in quantum mechanics. Phys. Rev. 31, 891-894 (1928)

13. Cushman, R.H., Bates, L.M.: Global Aspects of Classical Integrable Systems. Basel-Boston:Birkhäuser, 1997

14. Deser, S., Jackiw, R.: Classical and quantum scattering on a cone. Commun. Math. Phys. 118, 495-509 (1988)

15. Dimock, J.: Canonical quantization of Yang-Mills on a circle. Rev. Math. Phys. 8, 85-102 (1996)

16. Driver, B.K., Hall, B.C.: Yang-Mills theory and the Segal-Bargmann transform. Commun. Math. Phys. 201, 249-290 (1999)

17. Emmrich, C., Roemer, H.: Orbifolds as configuration spaces of systems with gauge symmetries. Commun. Math. Phys. 129, 69-94 (1990)

18. Fischer, E., Rudolph, G., Schmidt, M.: A lattice gauge model of singular Marsden-Weinstein reduction Part I. Kinematics. J. Geom. Phys. 57, 1193-1213 (2007)

19. Florentino, C.A., Mourão, J.M., Nunes, J.: Coherent state transforms and vector bundles on elliptic curves. J. Funct. Anal. 204, 355-398 (2003)

20. Goresky, M., MacPherson, R.: Stratified Morse theory. Berlin-Heidelberg, New York: Springer, 1988

21. Hall, B.C.: The Segal-Bargmann "coherent state" transform for compact Lie groups. J. Funct. Anal. 122, 103-151 (1994)

22. Hall, B.C.: The inverse Segal-Bargmann transform for compact Lie groups. J. Funct. Anal. 143, 98-116 (1997)

23. Hall, B.C.: Phase space bounds for quantum mechanics on a compact Lie group. Commun. Math. Phys. 184, 233-250 (1997)

24. Hall, B.C.: Coherent states and the quantization of 1+1-dimensional Yang-Mills theory. Rev. Math. Phys. 13, 1281-1306 (2001)

25. Hall, B.C.: Geometric quantization and the generalized Segal-Bargmann transform for Lie groups of compact type. Commun. Math. Phys. 226, 233-268 (2002)

26. Hall, B.C., Mitchell, J.J.: The Segal-Bargmann transform for noncompact symmetric spaces of the complex type. J. Funct. Anal. 227, 338-371 (2005)

27. Helgason, S.: Groups and geometric analysis. Integral geometry, invariant differential operators, and spherical functions. London-New York:Academic Press, 1984

28. Hetrick, J.E.: Canonical quantization of two-dimensional gauge fields. Int. J. Mod. Phys. A 9, 3153-3178 (1994)

29. Huebschmann, J.: Poisson geometry of flat connections for SU(2)-bundles on surfaces. Math. Z. 221, 243-259 (1996)

30. Huebschmann, J.: Symplectic and Poisson structures of certain moduli spaces. Duke Math. J. 80, 737-756 (1995)

31. Huebschmann, J.: Kähler spaces, nilpotent orbits, and singular reduction. Memoirs of the AMS 172 (814), Providence R.I.:Amer. Math. Soc., 2004

32. Huebschmann, J.: Kähler quantization and reduction. J. Reine. Angew. Math. 591, 75-109 (2006)

33. Huebschmann, J.: Stratified Kähler structures on adjoint quotients. Diff. Geom. Appl. 26, 704-731 (2008)

34. Huebschmann, J.: Singular Poisson-Kähler geometry of certain adjoint quotients, In: Proceedings, The mathematical legacy of C. Ehresmann, Bedlewo, 2005, Banach Center Publications 76, 325-347 (2007)

35. Huebschmann, J.: Kirillov's character formula, the holomorphic Peter-Weyl theorem, and the Blattner-Kostant-Sternberg pairing. J. Geom. Phys. 58, 833-848 (2008) 
36. Jarvis, P.D., Kijowski, J., Rudolph, G.: On the structure of the observable algebra of QCD on the lattice. J. Phys. A 38, 5359-5377 (2005)

37. Kay, B.S., Studer, U.M.: Boundary conditions for quantum mechanics on cones and fields around cosmic strings. Commun. Math. Phys. 139, 103-139 (1991)

38. Kijowski, J., Rudolph, G.: On the Gauss law and global charge for quantum chromodynamics. J. Math. Phys. 43, 1796-1808 (2002)

39. Kijowski, J., Rudolph, G.: Charge superselection sectors for qcd on the lattice. J. Math. Phys. 46, 032303 (2005)

40. Kijowski, J., Rudolph, G., Śliwa, C.: On the structure of the observable algebra for QED on the lattice. Lett. Math. Phys. 43, 99-308 (1998)

41. Kijowski, J., Rudolph, G., Śliwa, C.: Charge superselection sectors for scalar QED on the lattice. Ann. Henri. Poincaré. 4, 1137-1167 (2003)

42. Kijowski, J., Rudolph, G., Thielmann, A.: Algebra of observables and charge superselection sectors for QED on the lattice. Commun. Math. Phys. 188, 535-564 (1997)

43. Landsman, N.P.: Mathematical topics between classical and quantum mechanics. Berlin-Heidelberg, New York: Springer, 1998

44. Landsman, N.P., Wren, K.K.: Constrained quantization and $\theta$-angles. Nucl. Phys. B. 502, 537-560 (1997)

45. Landsman, N.P., Wren, K.K.: Hall's coherent states, the Cameron-Martin theorem, and the quantization of Yang-Mills theory on a circle. http://arxiv.org/list/math-ph/9812012, 1998

46. McLachlan, N.W.: Theory and Application of Mathieu Functions. New York:Dover Publications, 1964

47. Meixner J., Schaefke W.: Mathieusche Funktionen und Sphäroidfunktionen. Grundlehren Bd. 71. BerlinHeidelberg, New York: Springer, 1954

48. Nelson, E.: Analytic vectors. Ann. of Math. 70, 572-615 (1959)

49. Pradhan, T., Khare, A.V.: Plane pendulum in quantum mechanics. Amer. J. Phys. 41, 59-66 (1973)

50. Schwarz, G.W.: Smooth functions invariant under the action of a compact Lie group. Topology 14, 63-68 (1975)

51. Śniatycki, J.: Geometric quantization and quantum mechanics. Applied Mathematical Sciences 30, Berlin-Heidelberg, New York: Springer, 1980

52. Stein, E.M.: Topics in harmonic analysis related to the Littlewood-Paley theory. Annals of Mathematics Studies, No 63. Princeton, NJ:Princeton University Press, 1970

53. Taylor, J.: The Iwasawa decomposition and limiting behaviour of Brownian motion on symmetric spaces of non-compact type. Cont. Math. 73, 303-331 (1988)

54. Thiemann, T.: Gauge field theory coherent states (GCS). I. General properties. Class. Quant. Grav. 18, 2025-2064 (2001)

55. Thiemann, T., Winkler, O.: Gauge field theory coherent states (GCS). II. Peakedness properties. Class. Quant. Grav. 18, 2561-2636 (2001)

56. Woodhouse, N.M.J.: Geometric quantization. Oxford:Clarendon Press, 1991

57. Wren, K.K.: Quantization of constrained systems with singularities using Rieffel induction. J. Geom. Phys. 24, 173-202 (1998)

58. Wren, K.K.: Constrained quantisation and $\theta$-angles II. Nucl. Phys. B 521, 471-502 (1998)

Communicated by A. Connes 